\title{
Boundedness of maximal operator, Hardy operator and Sobolev's inequalities on non-homogeneous central Herz-Morrey-Musielak-Orlicz spaces
}

\author{
Fumi-Yuki Maeda, Yoshihiro Mizuta, Takao Ohno and Tetsu Shimomura \\ (Received December 25, 2019) \\ (Revised January 12, 2021)
}

\begin{abstract}
Our aim in this paper is to deal with the boundedness of the HardyLittlewood maximal operator and the Hardy operator on non-homogeneous central Herz-Morrey-Musielak-Orlicz spaces and to establish a generalization of Sobolev's inequalities for Riesz potentials of functions in such spaces.
\end{abstract}

\section{Introduction}

Let $\mathbf{R}^{N}$ be the Euclidean space and let $B(x, r)$ denote the open ball centered at $x \in \mathbf{R}^{N}$ with radius $r>0$.

In harmonic analysis, the maximal operator is a classical tool when studying Sobolev functions and partial differential equations. This also plays a central role in the study of differentiation, singular integrals, smoothness of functions and so on (see $[8,28,50]$, etc.). It is well known that the maximal operator is bounded on the Lebesgue space $L^{p}\left(\mathbf{R}^{N}\right)$ if $p>1$ (see [50]). The boundedness of the maximal operator was studied on Morrey spaces in [11, 42], on Orlicz-Morrey spaces in [44], and also on non-homogeneous Herz spaces in [29]. For Morrey spaces, which were introduced to estimate solutions of partial differential equations, we refer to $[40,46]$.

One of the important applications of the boundedness of the maximal operator is Sobolev's inequality; in classical Lebesgue spaces, we know Sobolev's inequality:

$$
\left\|I_{\alpha} f\right\|_{L^{p^{*}\left(\mathbf{R}^{N}\right)}} \leq C\|f\|_{L^{p}\left(\mathbf{R}^{N}\right)}
$$

for $f \in L^{p}\left(\mathbf{R}^{N}\right), 0<\alpha<N$ and $1<p<N / \alpha$, where $I_{\alpha}$ is the Riesz kernel of order $\alpha$ and $1 / p^{*}=1 / p-\alpha / N$ (see, e.g. [2, Theorem 3.1.4]). Sobolev's inequal-

The third author is supported by Grant-in-Aid for Scientific Research(C), No. 19K03586.

2010 Mathematics Subject Classification. Primary 31B15, 46E35.

Key words and phrases. Maximal operator, non-homogeneous central Herz-Morrey-MusielakOrlicz spaces, Riesz potentials, Sobolev's inequality. 
ity for Morrey spaces was given by D. R. Adams [1] (also [11, 42]), and then the result was extended to Orlicz-Morrey spaces in [43]. See also [29] for non-homogeneous Herz spaces and [20] for non-homogeneous central Morrey spaces. For local Morrey-type spaces, we refer the reader to $[9,10]$ and so on.

Variable exponent Lebesgue spaces and Sobolev spaces were introduced to discuss nonlinear partial differential equations with non-standard growth condition. For a survey, see $[15,19]$. The boundedness of the maximal and Riesz potential operators were studied for variable exponent Lebesgue spaces $L^{p(\cdot)}$ (see [16, 17, 18]), variable exponent Morrey spaces (see [4, 22, 23, 34, 39]), Herz spaces with variable exponents (see [3, 27, 47]), local variable exponent Morrey type spaces (see $[23,24]$ ) and non-homogeneous central Morrey spaces of variable exponent (see [38]).

Recently, the boundedness of the maximal and Riesz potential operators were studied for Herz-Morrey spaces with variable exponents (see [35, 36]) and non-homogeneous central Herz-Morrey-Orlicz spaces in the constant exponent case (see [37]).

Let $\Omega$ be a measurable set in $\mathbf{R}^{N}$. Given a general function $\Phi(x, t)$ satisfying certain conditions, we consider the associated Musielak-Orlicz space (cf. [41])

$$
L^{\Phi}(\Omega)=\left\{f \in L_{l o c}^{1}(\Omega) ; \int_{\Omega} \Phi(y,|f(y)|) d y<\infty\right\}
$$

which is a Banach space with respect to the norm

$$
\|f\|_{L^{\Phi}(\Omega)}=\inf \left\{\lambda>0 ; \int_{\Omega} \bar{\Phi}(y,|f(y)| / \lambda) d y \leq 1\right\}
$$

(see Section 2 for the definitions of $\Phi$ and $\bar{\Phi}$ ). For the recent development of the theory of PDEs in Musielak-Orlicz spaces and Herz spaces with variable exponents, we refer to $[7,12,25,48]$ and so on. Let $\omega(r):(0, \infty) \rightarrow(0, \infty)$ be almost monotone on $(0, \infty)$ satisfying the doubling condition. Let $0<q<\infty$. Given $\Phi(x, t)$ and $\omega(r)$, we denote by $\mathscr{H}^{\Phi, q, \omega}\left(\mathbf{R}^{N}\right)$ the class of locally integrable functions $f$ on $\mathbf{R}^{N}$ satisfying

$$
\|f\|_{\mathscr{H}^{\Phi, q, \omega}\left(\mathbf{R}^{N}\right)}=\|f\|_{L^{\Phi}(B(0,2))}+\left(\int_{1}^{\infty}\left(\omega(r)\|f\|_{L^{\Phi}(A(0, r))}\right)^{q} \frac{d r}{r}\right)^{1 / q}<\infty,
$$

where $A(0, r)=B(0,2 r) \backslash B(0, r)$. The space $\mathscr{H}^{\Phi, q, \omega}\left(\mathbf{R}^{N}\right)$ is referred to as a non-homogeneous central Herz-Morrey-Musielak-Orlicz space (see Section 2).

Our first aim in this paper is to study the boundedness of the maximal operator on non-homogeneous central Herz-Morrey-Musielak-Orlicz spaces 
$\mathscr{H}^{\Phi, q, \omega}\left(\mathbf{R}^{N}\right)$ (Theorem 1 below), as an extension of $[36,37]$. To this end, we apply the boundedness of the Hardy-Littlewood maximal operator on $L^{\Phi}$ given in [30]. The case when $q=\infty$ was treated in [45], as an extension of [35].

Next we study the boundedness of the Hardy operators $\hat{H}_{\beta}^{\infty}$ and $\hat{H}_{\beta}^{0}$ on $\mathscr{H}^{\Phi, q, \omega}\left(\mathbf{R}^{N}\right)$ (Theorems 2 and 3 below). See Section 4 for the definitions of $\hat{H}_{\beta}^{\infty}$ and $\hat{H}_{\beta}^{0}$.

As an application of the boundedness of the maximal operator, we establish Sobolev's inequality for Riesz potentials $I_{\alpha} f$ of functions in $\mathscr{H}^{\Phi, q, \omega}\left(\mathbf{R}^{N}\right)$ (Theorem 4 below), as an extension of $[36,37]$. When $q=\infty$, we refer to $[35,45]$.

Further, we discuss Sobolev's inequality for generalized Riesz potentials $I_{\alpha, k} f$ of functions in $\mathscr{H}^{\Phi, q, \omega}\left(\mathbf{R}^{N}\right)$ (Theorem 5 below), as an extension of [35, 36, 37]. See Section 6 for the definition of $I_{\alpha, k} f$.

In Section 7, in connection with the study in [21, 24], we investigate the space $\mathscr{H}^{\Phi, q, \omega}\left(\mathbf{R}^{N}\right)$ and its complementary space $\frac{\mathscr{H}}{\Phi, q, \omega}\left(\mathbf{R}^{N}\right)$.

In Section 8, we treat the case $q$ is variable.

Throughout this paper, let $C$ denote various positive constants independent of the variables in question. The symbol $g \sim h$ means that $C^{-1} h \leq g \leq C h$ for some constant $C>0$.

\section{Preliminaries}

We consider a function

$$
\Phi(x, t)=t \phi(x, t): \mathbf{R}^{N} \times[0, \infty) \rightarrow[0, \infty)
$$

satisfying the following conditions $(\Phi 1)-(\Phi 4)$ :

(Ф1) $\phi(\cdot, t)$ is measurable on $\mathbf{R}^{N}$ for each $t \geq 0$ and $\phi(x, \cdot)$ is continuous on $[0, \infty)$ for each $x \in \mathbf{R}^{N}$;

(Ф2) there exists a constant $A_{1} \geq 1$ such that

$$
A_{1}^{-1} \leq \phi(x, 1) \leq A_{1} \quad \text { for all } x \in \mathbf{R}^{N}
$$

(Ф3) $\phi(x, \cdot)$ is uniformly almost increasing; namely there exists a constant $A_{2} \geq 1$ such that

$$
\phi(x, t) \leq A_{2} \phi(x, s) \quad \text { for all } x \in \mathbf{R}^{N} \text { whenever } 0 \leq t<s ;
$$

(Ф4) there exists a constant $A_{3} \geq 1$ such that

$$
\phi(x, 2 t) \leq A_{3} \phi(x, t) \quad \text { for all } x \in \mathbf{R}^{N} \text { and } t>0 .
$$


Note that $(\Phi 2),(\Phi 3)$ and ( $\Phi 4)$ imply

$$
0<\inf _{x \in \mathbf{R}^{N}} \phi(x, t) \leq \sup _{x \in \mathbf{R}^{N}} \phi(x, t)<\infty
$$

for each $t>0$.

If $\Phi(x, \cdot)$ is convex for each $x \in \mathbf{R}^{N}$, then ( $\left.\Phi 3\right)$ holds with $A_{2}=1$; namely $\phi(x, \cdot)$ is non-decreasing for each $x \in \mathbf{R}^{N}$.

Let $\bar{\phi}(x, t)=\sup _{0 \leq s \leq t} \phi(x, s)$ and

$$
\bar{\Phi}(x, t)=\int_{0}^{t} \bar{\phi}(x, r) d r
$$

for $x \in \mathbf{R}^{N}$ and $t \geq 0$. Then $\bar{\Phi}(x, \cdot)$ is convex and

$$
\frac{1}{2 A_{3}} \Phi(x, t) \leq \bar{\Phi}(x, t) \leq A_{2} \Phi(x, t)
$$

for all $x \in \mathbf{R}^{N}$ and $t \geq 0$.

By $(\Phi 3)$, we see that

$$
\Phi(x, a t) \begin{cases}\leq A_{2} a \Phi(x, t) & \text { if } 0 \leq a \leq 1, \\ \geq A_{2}^{-1} a \Phi(x, t) & \text { if } a \geq 1\end{cases}
$$

We shall also consider the following conditions for $\Phi(x, t)$ : Let $p \geq 1$, $q \geq 1, \eta>0$ and $\tau>0$.

$(\Phi 3 ; 0 ; p) \quad t \mapsto t^{-p} \Phi(x, t)$ is uniformly almost increasing on $(0,1]$, namely there exists a constant $A_{2,0, p} \geq 1$ such that

$$
t_{1}^{-p} \Phi\left(x, t_{1}\right) \leq A_{2,0, p} t_{2}^{-p} \Phi\left(x, t_{2}\right) \quad \text { for all } x \in \mathbf{R}^{N} \text { whenever } 0<t_{1}<t_{2} \leq 1 ;
$$

$(\Phi 3 ; \infty ; q) \quad t \mapsto t^{-q} \Phi(x, t)$ is uniformly almost increasing on $[1, \infty)$, namely there exists a constant $A_{2, \infty, q} \geq 1$ such that

$$
t_{1}^{-q} \Phi\left(x, t_{1}\right) \leq A_{2, \infty, q} t_{2}^{-q} \Phi\left(x, t_{2}\right) \quad \text { for all } x \in \mathbf{R}^{N} \text { whenever } 1 \leq t_{1}<t_{2} ;
$$

$(\Phi 5 ; \eta) \quad$ for every $\gamma>0$, there exists a constant $B_{\gamma, \eta} \geq 1$ such that

$$
\Phi(x, t) \leq B_{\gamma, \eta} \Phi(y, t)
$$

whenever $x, y \in \mathbf{R}^{N},|x-y| \leq \gamma t^{-\eta}$ and $t \geq 1$;

$(\Phi 6 ; \tau) \quad$ there exist a function $g$ on $\mathbf{R}^{N}$ and a constant $B_{\infty} \geq 1$ such that $0 \leq g(x) \leq 1$ for all $x \in \mathbf{R}^{N}, g \in L^{\tau}\left(\mathbf{R}^{N}\right)$ and

$$
B_{\infty}^{-1} \Phi(x, t) \leq \Phi\left(x^{\prime}, t\right) \leq B_{\infty} \Phi(x, t)
$$

whenever $x, x^{\prime} \in \mathbf{R}^{N},\left|x^{\prime}\right| \geq|x|$ and $g(x) \leq t \leq 1$. 
Note that $(\Phi 3 ; 0 ; 1)+(\Phi 3 ; \infty ; 1)=(\Phi 3)$. If $\Phi(x, t)$ satisfies $(\Phi 3 ; 0 ; p)$, then it satisfies $\left(\Phi 3 ; 0 ; p^{\prime}\right)$ for $1 \leq p^{\prime} \leq p$; if $\Phi(x, t)$ satisfies $(\Phi 3 ; \infty ; q)$, then it satisfies $\left(\Phi 3 ; \infty ; q^{\prime}\right)$ for $1 \leq q^{\prime} \leq q$.

If $\Phi(x, t)$ satisfies $(\Phi 3 ; 0 ; p)$, then

$$
\Phi(x, t) \leq A_{1} A_{2,0, p} t^{p} \quad \text { for } 0 \leq t \leq 1 ;
$$

if $\Phi(x, t)$ satisfies $(\Phi 3 ; \infty ; q)$, then

$$
\Phi(x, t) \geq\left(A_{1} A_{2, \infty, q}\right)^{-1} t^{q} \quad \text { for } t \geq 1 .
$$

If $\Phi(x, t)$ satisfies $(\Phi 5 ; \eta)$, then it satisfies $\left(\Phi 5 ; \eta^{\prime}\right)$ for all $\eta^{\prime} \geq \eta$; if $\Phi(x, t)$ satisfies $(\Phi 6 ; \tau)$, then it satisfies $\left(\Phi 6 ; \tau^{\prime}\right)$ for all $\tau^{\prime} \geq \tau$.

In the following examples, we use the notation

$$
f^{-}:=\inf _{x \in \mathbf{R}^{N}} f(x) \quad \text { and } \quad f^{+}:=\sup _{x \in \mathbf{R}^{N}} f(x)
$$

for a measurable function $f$ on $\mathbf{R}^{N}$.

EXAmple 1. Let $p_{i}(\cdot), i=1,2$ and $q_{i, j}(\cdot), j=1, \ldots, k_{i}$, be real valued measurable functions on $\mathbf{R}^{N}$ such that $p_{i}^{-}>1$ and $q_{i, j}^{-}>-\infty, i=1,2, j=$ $1, \ldots, k_{i}$.

Set $L_{c}(t)=\log (c+t)$ for $c>1$ and $t \geq 0, \quad L_{c}^{(1)}(t)=L_{c}(t), \quad L_{c}^{(j+1)}(t)=$ $L_{c}\left(L_{c}^{(j)}(t)\right)$. Let

$$
\Phi(x, t)= \begin{cases}t^{p_{1}(x)} \prod_{j=1}^{k_{1}}\left(L_{e-1}^{(j)}(1 / t)\right)^{-q_{1, j}(x)} & \text { if } 0 \leq t \leq 1, \\ t^{p_{2}(x)} \prod_{j=1}^{k_{2}}\left(L_{e-1}^{(j)}(t)\right)^{q_{2, j}(x)} & \text { if } t \geq 1 .\end{cases}
$$

Then, $\Phi(x, t)$ satisfies $(\Phi 1),(\Phi 2)$ and $(\Phi 3)$. It satisfies $(\Phi 3 ; 0 ; p)$ for $1 \leq p<$ $p_{1}^{-}$in general and for $1 \leq p \leq p_{1}^{-}$in case $q_{1, j}^{-} \geq 0$ for all $j=1, \ldots, k_{1}$; it satisfies $(\Phi 3 ; \infty ; q)$ for $1 \leq q<p_{2}^{-}$in general and for $1 \leq q \leq p_{2}^{-}$in case $q_{2, j}^{-} \geq 0$ for all $j=1, \ldots, k_{2}$.

Moreover, we see that $\Phi(x, t)$ satisfies $(\Phi 5 ; \eta)$ for every $\eta>0$ if $p_{2}(\cdot)$ is log-Hölder continuous, namely

$$
\left|p_{2}(x)-p_{2}(y)\right| \leq \frac{C_{p}}{L_{e}(1 /|x-y|)} \quad\left(x, y \in \mathbf{R}^{N}\right)
$$

with a constant $C_{p} \geq 0$ and $q_{2, j}(\cdot)$ is $(j+1)$-log-Hölder continuous, namely

$$
\left|q_{2, j}(x)-q_{2, j}(y)\right| \leq \frac{C_{j}}{L_{e}^{(j+1)}(1 /|x-y|)} \quad\left(x, y \in \mathbf{R}^{N}\right)
$$

with constants $C_{j} \geq 0$, for each $j=1, \ldots, k_{2}$. 
Finally, we see that $\Phi(x, t)$ satisfies $(\Phi 6 ; \tau)$ for every $\tau>0$ with $g(x)=$ $(1+|x|)^{-(N+1) / \tau}$ if $p_{1}(\cdot)$ is log-Hölder continuous at $\infty$, namely

$$
\left|p_{1}(x)-p_{1}\left(x^{\prime}\right)\right| \leq \frac{C_{p, \infty}}{L_{e}(|x|)}
$$

whenever $\left|x^{\prime}\right| \geq|x|\left(x, x^{\prime} \in \mathbf{R}^{N}\right)$ with a constant $C_{p, \infty} \geq 0$, and $q_{1, j}(\cdot)$ is $(j+1)$ log-Hölder continuous at $\infty$, namely

$$
\left|q_{1, j}(x)-q_{1, j}\left(x^{\prime}\right)\right| \leq \frac{C_{j}^{\prime}}{L_{e}^{(j+1)}(|x|)}
$$

whenever $\left|x^{\prime}\right| \geq|x|\left(x, x^{\prime} \in \mathbf{R}^{N}\right)$ with a constant $C_{j}^{\prime} \geq 0$, for each $j=1, \ldots, k_{1}$. In fact, if $(1+|x|)^{-(N+1) / \tau}<t \leq 1$, then $t^{-\left|p_{1}(x)-p_{1}\left(x^{\prime}\right)\right|} \leq e^{(N+1) C_{p, \infty} / \tau}$ for $\left|x^{\prime}\right| \geq|x|$ and $\left(L_{e-1}^{(j)}(1 / t)\right)^{\left|q_{1, j}(x)-q_{1, j}\left(x^{\prime}\right)\right|} \leq C\left(N, C_{j}^{\prime}\right)$ for $\left|x^{\prime}\right| \geq|x|$.

The following example shows that our conditions are satisfied by the double phase functional with variable exponents.

EXAMPLE 2. Regarding regularity theory of differential equations, Baroni, Colombo and Mingione [5, 6, 7, 13, 14] have studied the double phase functional

$$
\Phi(x, t)=t^{p}+a(x) t^{q},
$$

where $1<p<q, a(\cdot)$ is non-negative, bounded and Hölder continuous of order $\theta \in(0,1]$. In [31], we studied the double phase functional with variable exponents:

$$
\Phi(x, t)=t^{p(x)}+a(x) t^{q(x)}, \quad x \in \mathbf{R}^{N}, t \geq 0,
$$

where $p(\cdot)$ and $q(\cdot)$ are real valued functions on $\mathbf{R}^{N}$ such that $p(x)<q(x)$ for $x \in \mathbf{R}^{N}, a(\cdot)$ is non-negative, bounded and Hölder continuous of order $\theta \in(0,1]$. This $\Phi(x, t)$ satisfies $(\Phi 1),(\Phi 2),(\Phi 3),\left(\Phi 3 ; 0 ; p^{-}\right),\left(\Phi 3 ; \infty ; p^{-}\right)$and $(\Phi 5 ; \eta)$ for $\eta \geq \sup _{\{x: a(x)>0\}}(q(x)-p(x)) / \theta$ if $1 \leq p^{-} \leq p^{+}<\infty, 1 \leq q^{-} \leq$ $q^{+}<\infty, p(\cdot)$ and $q(\cdot)$ are log-Hölder continuous. Further it satisfies $(\Phi 6 ; \tau)$ with $g(x)=(1+|x|)^{-(N+1) / \tau}$ for every $\tau>0$ if $p(\cdot)$ is log-Hölder continuous at $\infty$. See [31] for details.

Let $\Omega$ be a measurable set in $\mathbf{R}^{N}$. From now on, we assume that $\Phi(x, t)$ satisfies $(\Phi 1),(\Phi 2),(\Phi 3)$ and $(\Phi 4)$. Then the associated Musielak-Orlicz space

$$
L^{\Phi}(\Omega)=\left\{f \in L_{l o c}^{1}(\Omega) ; \int_{\Omega} \Phi(y,|f(y)|) d y<\infty\right\}
$$


is a Banach space with respect to the norm

$$
\|f\|_{L^{\Phi}(\Omega)}=\inf \left\{\lambda>0 ; \int_{\Omega} \bar{\Phi}(y,|f(y)| / \lambda) d y \leq 1\right\}
$$

(cf. [41]).

REMARK 1. The Musielak-Orlicz spaces $L^{\Phi}(\Omega)$ include the following spaces:

- Orlicz spaces defined by Young functions satisfying the doubling condition;

- variable exponent Lebesgue spaces.

REMARK 2. The dominated convergence theorem and ( $\Phi 4)$ yield

$$
\int_{\Omega} \bar{\Phi}\left(y, \frac{|f(y)|}{\|f\|_{L^{\Phi}(\Omega)}}\right) d y=1
$$

We consider a function $\omega(r):(0, \infty) \rightarrow(0, \infty)$ satisfying the following conditions $(\omega 1)$ and $(\omega 2)$ :

$(\omega 1) \omega(\cdot)$ is almost monotone on $(0, \infty)$; that is, $\omega(\cdot)$ is almost increasing on $(0, \infty)$ or $\omega(\cdot)$ is almost decreasing on $(0, \infty)$; namely there exists a constant $c_{1}>0$ such that

$$
\omega(r) \leq c_{1} \omega(s) \quad \text { for all } 0<r<s
$$

or

$$
\omega(s) \leq c_{1} \omega(r) \quad \text { for all } 0<r<s
$$

respectively;

( $\omega 2) \omega(\cdot)$ is doubling on $(0, \infty)$; that is, there exists a constant $c_{2}>1$ such that

$$
c_{2}^{-1} \omega(r) \leq \omega(2 r) \leq c_{2} \omega(r) \quad \text { for all } r>0 .
$$

Let $0<q<\infty$. Given $\Phi(x, t)$ and $\omega(r)$ as above, we denote by $\mathscr{H}^{\Phi, q, \omega}\left(\mathbf{R}^{N}\right)$ the class of locally integrable functions $f$ on $\mathbf{R}^{N}$ satisfying

$$
\|f\|_{\mathscr{H}^{\Phi, q, \omega}\left(\mathbf{R}^{N}\right)}=\|f\|_{L^{\Phi}(B(0,2))}+\left(\int_{1}^{\infty}\left(\omega(r)\|f\|_{L^{\Phi}(A(0, r))}\right)^{q} \frac{d r}{r}\right)^{1 / q}<\infty,
$$

where $A(0, r)=B(0,2 r) \backslash B(0, r)$. The space $\mathscr{H}^{\Phi, q, \omega}\left(\mathbf{R}^{N}\right)$ is referred to as a non-homogeneous central Herz-Morrey-Musielak-Orlicz space.

REMARK 3. The non-homogeneous central Herz-Morrey-Musielak-Orlicz spaces $\mathscr{H}^{\Phi, q, \omega}\left(\mathbf{R}^{N}\right)$ include the following spaces: 
- non-homogeneous Herz spaces introduced in [26];

- local Morrey-type spaces introduced in [9];

- non-homogeneous central Herz-Morrey-Orlicz spaces introduced in [37] where $\Phi(x, t)=\Phi(t)$;

- non-homogeneous central Herz-Morrey spaces with variable exponents introduced in [36] where $\Phi(x, t)=t^{p(x)}$.

LeMma 1. For $1 / 2<a<1<b<2$ with $2 a \geq b$, there exists a constant $C>0$ such that

$$
\int_{a t}^{b t}\left(\omega(r)\|f\|_{L^{\Phi}(A(0, r))}\right)^{q} \frac{d r}{r} \geq C\left(\omega(t)\|f\|_{L^{\Phi}(A(0, t))}\right)^{q}
$$

for all $t>0$.

Proof. For $1 / 2<a<1<b<2$ with $2 a \geq b$, we have

$$
\int_{a t}^{t}\left(\omega(r)\|f\|_{L^{\Phi}(A(0, r))}\right)^{q} \frac{d r}{r} \geq C\left(\omega(t)\|f\|_{L^{\Phi}(B(0,2 a t) \backslash B(0, t))}\right)^{q}
$$

and

$$
\int_{t}^{b t}\left(\omega(r)\|f\|_{L^{\Phi}(A(0, r))}\right)^{q} \frac{d r}{r} \geq C\left(\omega(t)\|f\|_{L^{\Phi}(B(0,2 t) \backslash B(0, b t))}\right)^{q},
$$

so that we obtain

$$
\begin{aligned}
(\omega(t) & \left.\|f\|_{L^{\Phi}(A(0, t))}\right)^{q} \\
\leq & \left(\omega(t)\|f\|_{L^{\Phi}(B(0,2 t) \backslash B(0, b t))}+\omega(t)\|f\|_{L^{\Phi}(B(0,2 a t) \backslash B(0, t))}\right)^{q} \\
& \leq C\left\{\int_{t}^{b t}\left(\omega(r)\|f\|_{L^{\Phi}(A(0, r))}\right)^{q} \frac{d r}{r}+\int_{a t}^{t}\left(\omega(r)\|f\|_{L^{\Phi}(A(0, r))}\right)^{q} \frac{d r}{r}\right\} \\
& =C \int_{a t}^{b t}\left(\omega(r)\|f\|_{L^{\Phi}(A(0, r))}\right)^{q} \frac{d r}{r} .
\end{aligned}
$$

Lemma 2. For a bounded measurable set $\Omega$, there exist constants $C_{\Omega}$ and $C_{\Omega}^{\prime}$ such that

$$
\int_{\Omega}|f(x)| d x \leq C_{\Omega}\|f\|_{L^{\Phi}(\Omega)} \leq C_{\Omega}^{\prime}\|f\|_{\mathscr{H}^{\Phi, q, \omega}\left(\mathbf{R}^{N}\right)}
$$

for all $f \in \mathscr{H}^{\Phi, q, \omega}\left(\mathbf{R}^{N}\right)$.

Proof. If $\|f\|_{L^{\Phi}(\Omega)} \leq 1$, then

$$
\int_{\Omega}|f(x)| d x \leq|\Omega|+2 A_{1} A_{3} \int_{\Omega} \bar{\Phi}(x,|f(x)|) d x \leq|\Omega|+2 A_{1} A_{3}
$$


by $(\Phi 2)$, convexity of $\bar{\Phi}(x, \cdot)$ and (1), where $|\Omega|$ denotes the Lebesgue measure of $\Omega$. This shows the first inequality in (3).

Next, suppose $f \in \mathscr{H}^{\Phi, q, \omega}\left(\mathbf{R}^{N}\right)$ and $\Omega \subset B\left(0,2^{k}\right)(k \geq 1)$. Then

$$
\begin{aligned}
\|f\|_{L^{\Phi}(\Omega)} & \leq\|f\|_{L^{\Phi}(B(0,2))}+\sum_{j=1}^{k-1}\|f\|_{L^{\Phi}\left(A\left(0,2^{j}\right)\right)} \\
& \leq\|f\|_{L^{\Phi}(B(0,2))}+C_{k} \sum_{j=1}^{k-1} \omega\left(2^{j}\right)\|f\|_{L^{\Phi}\left(A\left(0,2^{j}\right)\right)},
\end{aligned}
$$

where $C_{k}^{-1}=\inf _{2 \leq r \leq 2^{k-1}} \omega(r)>0$. Then, using Lemma 1, we obtain the second inequality in (3).

Lemma 3 (cf. [30, Lemma 5.1]). Let $F(x, t)$ be a positive function on $\mathbf{R}^{N} \times(0, \infty)$ satisfying the following conditions:

(F1) $F(x, \cdot)$ is strictly increasing and continuous on $(0, \infty)$ for each $x \in \mathbf{R}^{N}$;

(F2) there exists a constant $K_{1} \geq 1$ such that

$$
K_{1}^{-1} \leq F(x, 1) \leq K_{1} \quad \text { for all } x \in \mathbf{R}^{N}
$$

(F3) $t \mapsto t^{-\varepsilon} F(x, t)$ is uniformly almost increasing for $\varepsilon>0$; namely there exists a constant $K_{2} \geq 1$ such that

$$
t^{-\varepsilon} F(x, t) \leq K_{2} s^{-\varepsilon} F(x, s) \quad \text { for all } x \in \mathbf{R}^{N} \text { whenever } 0<t<s ;
$$

(F4) there exists a constant $K_{3}>1$ such that

$$
F(x, 2 t) \leq K_{3} F(x, t) \quad \text { for all } x \in \mathbf{R}^{N} \text { and } t>0 .
$$

Let $F^{-1}(x, \cdot)$ be the inverse function of $F(x, \cdot)$. Then:

(1) $F^{-1}(x, \cdot)$ is strictly increasing.

(2)

$$
F^{-1}(x, \lambda t) \leq\left(K_{2} \lambda\right)^{1 / \varepsilon} F^{-1}(x, t)
$$

for all $x \in \mathbf{R}^{N}, t>0$ and $\lambda \geq 1$.

(3)

$$
F^{-1}(x, \lambda t) \leq 2 \lambda^{1 / \log _{2} K_{3}} F^{-1}(x, t)
$$

for all $x \in \mathbf{R}^{N}, t>0$ and $0<\lambda<1$.

(4)

$$
\min \left\{1,\left(\frac{t}{K_{1} K_{2}}\right)^{1 / \varepsilon}\right\} \leq F^{-1}(x, t) \leq \max \left\{1,\left(K_{1} K_{2} t\right)^{1 / \varepsilon}\right\}
$$

for all $x \in \mathbf{R}^{N}$ and $t>0$. 
REMARK 4. $\quad F(x, t)=\bar{\Phi}(x, t)$ satisfies (F1), (F2), (F3) and (F4) with $K_{1}=$ $A_{1} \max \left\{A_{2}, 2 A_{3}\right\}, K_{2}=1, K_{3}=2 A_{3}$ and $\varepsilon=1$.

We also consider a convex function $\Phi_{\infty}(t)=t \phi_{\infty}(t):[0, \infty) \rightarrow[0, \infty)$ such that $\phi_{\infty}(t)>0$ for $t>0, \phi_{\infty}(t)$ is increasing on $[0, \infty)$ and satisfies the doubling condition and

$\left(\Phi_{\infty} 1\right)$ there exists a constant $Q \geq 1$ such that

$$
Q^{-1} \Phi(x, t) \leq \Phi_{\infty}(t) \leq Q \Phi(x, t) \quad \text { whenever } g(x) \leq t \leq 1
$$

for $g$ in condition $(\Phi 6 ; \tau)$.

REMARK 5. Note from $\left(\Phi_{\infty} 1\right)$ that for $c_{1}, c_{2}>0$, there exists a constant $Q \geq 1$ such that

$$
Q^{-1} \Phi(x, t) \leq \Phi_{\infty}(t) \leq Q \Phi(x, t) \quad \text { whenever } c_{1} g(x) \leq t \leq c_{2}
$$

for $g$ in condition $(\Phi 6 ; \tau)$.

Remark 6. Suppose $\Phi(x, t)$ satisfies $(\Phi 6 ; \tau)$. Set

$$
\Phi_{\infty}(t)=\limsup _{|x| \rightarrow \infty} \bar{\Phi}(x, t) \quad \text { and } \quad \phi_{\infty}(t)=\Phi_{\infty}(t) / t .
$$

Then note that $\phi_{\infty}(t)>0$ for $t>0, \phi_{\infty}(t)$ is increasing on $[0, \infty)$ and satisfies the doubling condition. Further, by $(\Phi 6 ; \tau)$, we find that $\Phi_{\infty}(t)$ satisfies $\left(\Phi_{\infty} 1\right)$.

We denote by $\chi_{E}$ the characteristic function of $E$ and by $\Phi_{\infty}^{-1}(t)$ the inverse of $\Phi_{\infty}(t)$.

LEMMA 4. Assume

$\left(\Phi_{\infty} 2\right)$ there exists a constant $Q \geq 1$ such that

$$
\Phi_{\infty}(g(x)) \leq Q(1+|x|)^{-N}
$$

for all $x \in \mathbf{R}^{N}$.

Then there is a constant $C>0$ such that

$$
\left\|\chi_{B(0, r)}\right\|_{L^{\Phi}\left(\mathbf{R}^{N}\right)} \leq C\left\{\Phi_{\infty}^{-1}\left(r^{-N}\right)\right\}^{-1}
$$

for all $r \geq 1$.

Proof. Note from $\left(\Phi_{\infty} 2\right)$ and Lemma 3 (2) that

$$
g(x) \leq C \Phi_{\infty}^{-1}\left((1+|x|)^{-N}\right) \leq C \Phi_{\infty}^{-1}(1)
$$

for all $x \in \mathbf{R}^{N}$. 
Let $R \geq 1 / 2$. We have by $(\Phi 3),(\Phi 4)$, Lemma $3(2)$ and $\left(\Phi_{\infty} 1\right)$

$$
\begin{aligned}
\int_{A(0, R)} \Phi\left(y, \Phi_{\infty}^{-1}\left(R^{-N}\right)\right) d y & \leq C \int_{A(0, R)} \Phi\left(y, \Phi_{\infty}^{-1}\left((1+|y|)^{-N}\right)\right) d y \\
& \leq C \int_{A(0, R)} \Phi_{\infty}\left(\Phi_{\infty}^{-1}\left((1+|y|)^{-N}\right)\right) d y \\
& \leq C \int_{A(0, R)}(1+|y|)^{-N} d y \\
& \leq C .
\end{aligned}
$$

Hence we obtain

$$
\left\|\chi_{A(0, R)}\right\|_{L^{\Phi}\left(\mathbf{R}^{N}\right)} \leq C\left\{\Phi_{\infty}^{-1}\left(R^{-N}\right)\right\}^{-1}
$$

for all $R \geq 1 / 2$.

Here note from Lemma 3 (4) that

$$
\Phi_{\infty}^{-1}\left(R^{-N}\right) \leq \max \left\{1, C R^{-N}\right\} \leq C,
$$

so that

$$
\max \left\{\left\{\Phi_{\infty}^{-1}\left(R^{-N}\right)\right\}^{-1}, 1\right\} \leq C\left\{\Phi_{\infty}^{-1}\left(R^{-N}\right)\right\}^{-1}
$$

for all $R \geq 1 / 2$.

Fix $r \geq 1$. Let $j_{0}$ be the largest integer such that $2^{-j_{0}+1} r \geq 1$. Now we see from Lemma $3(3)$ that $t \mapsto t^{-\varepsilon}\left\{\Phi_{\infty}^{-1}\left(t^{-N}\right)\right\}^{-1}$ is almost increasing on $(0, \infty)$ for some constant $\varepsilon>0$, so that

$$
\begin{aligned}
\left\|\chi_{B(0, r)}\right\|_{L^{\Phi}\left(\mathbf{R}^{N}\right)} & \leq \sum_{j=1}^{j_{0}}\left\|\chi_{A\left(0,2^{-j} r\right)}\right\|_{L^{\Phi}\left(\mathbf{R}^{N}\right)}+\left\|\chi_{B(0,1)}\right\|_{L^{\Phi}\left(\mathbf{R}^{N}\right)} \\
& \leq C\left\{\sum_{j=1}^{j_{0}}\left\{\Phi_{\infty}^{-1}\left(\left(2^{-j} r\right)^{-N}\right)\right\}^{-1}+1\right\} \\
& \leq C\left\{r^{-\varepsilon}\left\{\Phi_{\infty}^{-1}\left(r^{-N}\right)\right\}^{-1} \sum_{j=1}^{j_{0}}\left(2^{-j} r\right)^{\varepsilon}+1\right\} \\
& \leq C\left\{\Phi_{\infty}^{-1}\left(r^{-N}\right)\right\}^{-1},
\end{aligned}
$$

as required.

REMARK 7. If $g(x) \leq C(1+|x|)^{-N}$, then $\left(\Phi_{\infty} 2\right)$ holds by convexity of $\Phi_{\infty}$. 
REMARK 8. Let $\Phi(x, t)$ and $g(x)$ be as in Example 1. Then there exist constants $p_{1}(\infty)>1$ and $q_{1, j}(\infty) \in \mathbf{R}$ for $j=1, \ldots, k_{1}$ such that

$$
\lim _{|x| \rightarrow \infty} p_{1}(x)=p_{1}(\infty) \quad \text { and } \quad \lim _{|x| \rightarrow \infty} q_{1, j}(x)=q_{1, j}(\infty) .
$$

Set $\bar{\phi}_{\infty}(t)=\sup _{0 \leq s \leq t}\left\{s^{p_{1}(\infty)-1} \prod_{j=1}^{k_{1}}\left(L_{e-1}^{(j)}(1 / s)\right)^{-q_{1, j}(\infty)}\right\}$ and

$$
\Phi_{\infty}(t)=\int_{0}^{t} \bar{\phi}_{\infty}(r) \frac{d r}{r}
$$

Then $\Phi_{\infty}(t)$ satisfies $\left(\Phi_{\infty} 1\right)$ and $\left(\Phi_{\infty} 2\right)$ for $0<\tau<(N+1) p_{1}(\infty) / N$.

Lemma 5. Suppose that $\Phi_{\infty}(t)$ satisfies $\left(\Phi_{\infty} 2\right)$. Then there is a constant $C>0$ such that

$$
\frac{1}{|A(0, r)|} \int_{A(0, r)}|f(y)| d y \leq C \Phi_{\infty}^{-1}\left(r^{-N}\right)\|f\|_{L^{\Phi}(A(0, r))}
$$

when $r \geq 1$ and $\|f\|_{L^{\Phi}(A(0, r))}<\infty$.

Proof. Fix $r \geq 1$. Let $f$ be a nonnegative measurable function on $A(0, r)$ satisfying $\|f\|_{L^{\Phi}(A(0, r))} \leq 1$. Then we have by $(\Phi 3)$

$$
\begin{aligned}
& \frac{1}{|A(0, r)|} \int_{A(0, r)} f(y) d y \\
& \quad \leq \Phi_{\infty}^{-1}\left(r^{-N}\right)+\frac{A_{2}}{|A(0, r)|} \int_{A(0, r)} f(y) \frac{\varphi(y, f(y))}{\varphi\left(y, \Phi_{\infty}^{-1}\left(r^{-N}\right)\right)} d y \\
& \quad=\Phi_{\infty}^{-1}\left(r^{-N}\right)+\frac{A_{2} \Phi_{\infty}^{-1}\left(r^{-N}\right)}{|A(0, r)|} \int_{A(0, r)} \Phi(y, f(y))\left\{\Phi\left(y, \Phi_{\infty}^{-1}\left(r^{-N}\right)\right)\right\}^{-1} d y .
\end{aligned}
$$

Since

$$
g(y) \leq C \Phi_{\infty}^{-1}\left((1+|y|)^{-N}\right) \leq C \Phi_{\infty}^{-1}\left(r^{-N}\right) \leq C \Phi_{\infty}^{-1}(1)
$$

for all $y \in A(0, r)$ by $\left(\Phi_{\infty} 2\right)$, we have by $\left(\Phi_{\infty} 1\right)$

$$
\Phi\left(y, \Phi_{\infty}^{-1}\left(r^{-N}\right)\right) \geq C r^{-N}
$$

for all $y \in A(0, r)$. Hence we obtain

$$
\begin{aligned}
\frac{1}{|A(0, r)|} \int_{A(0, r)} f(y) d y & \leq \Phi_{\infty}^{-1}\left(r^{-N}\right)+C \Phi_{\infty}^{-1}\left(r^{-N}\right) \int_{A(0, r)} \Phi(y, f(y)) d y \\
& \leq C \Phi_{\infty}^{-1}\left(r^{-N}\right),
\end{aligned}
$$

as required. 


\section{Boundedness of the maximal operator}

For a locally integrable function $f$ on $\mathbf{R}^{N}$, the Hardy-Littlewood maximal function $M f$ is defined by

$$
M f(x)=\sup _{r>0} \frac{1}{|B(x, r)|} \int_{B(x, r)}|f(y)| d y .
$$

The mapping $f \mapsto M f$ is called the maximal operator.

By [31, Theorem 3.1], we have the following result.

Lemma 6. Suppose that $\Phi(x, t)$ satisfies $(\Phi 3 ; 0 ; p),(\Phi 3 ; \infty ; q),(\Phi 5 ; \eta)$ and $(\Phi 6 ; \tau)$ for $p>1, q>1, \eta>0$ and $\tau>0$ satisfying $\eta \leq q / N$ and $\tau \leq p$. Then the maximal operator $M$ is bounded from $L^{\Phi}\left(\mathbf{R}^{N}\right)$ into itself; namely, there is a constant $C>0$ such that

$$
\|M f\|_{L^{\Phi}\left(\mathbf{R}^{N}\right)} \leq C\|f\|_{L^{\Phi}\left(\mathbf{R}^{N}\right)}
$$

for all $f \in L^{\Phi}\left(\mathbf{R}^{N}\right)$.

For a nonnegative function $f \in L_{l o c}^{1}\left(\mathbf{R}^{N}\right)$ and a real number $\beta$, set

$$
H_{\beta}^{\infty} f(r)=r^{\beta} \int_{\mathbf{R}^{N} \backslash B(0,2 r)}|y|^{-N-\beta} f(y) d y .
$$

LEMMA 7. For a real number $\beta$, suppose that $\Phi_{\infty}(t)$ satisfies $\left(\Phi_{\infty} 2\right)$ and $\left(\Phi_{\infty} \omega 1 ; \beta\right) \quad t^{\varepsilon_{1}-\beta} \omega(t)^{-1} \Phi_{\infty}^{-1}\left(t^{-N}\right)$ is almost decreasing in $[1, \infty)$ for some $\varepsilon_{1}>0$. If $0<\varepsilon<\varepsilon_{1}$, then there exists a constant $C>0$ such that

$$
H_{\beta}^{\infty} f(r) \leq C r^{\varepsilon} \omega(r)^{-1} \Phi_{\infty}^{-1}\left(r^{-N}\right)\left(\int_{r}^{\infty}\left(t^{-\varepsilon} \omega(t)\|f\|_{L^{\Phi}(A(0, t))}\right)^{q} \frac{d t}{t}\right)^{1 / q}
$$

for all $r \geq 1$ and nonnegative functions $f \in L_{\text {loc }}^{1}\left(\mathbf{R}^{N}\right)$.

Proof. Let $f \in L_{\text {loc }}^{1}\left(\mathbf{R}^{N}\right)$ be a nonnegative function on $\mathbf{R}^{N}$. Let $r \geq 1$ and $0<\varepsilon<\varepsilon_{1}$.

First we consider the case $1<q<\infty$. Then we have by Lemma 5 and Hölder's inequality

$$
\begin{aligned}
H_{\beta}^{\infty} f(r) & =r^{\beta} \sum_{j=1}^{\infty} \int_{A\left(0,2^{j} r\right)}|y|^{-N-\beta} f(y) d y \\
& \leq C r^{\beta} \sum_{j=1}^{\infty}\left(2^{j} r\right)^{-\beta} \frac{1}{\left|A\left(0,2^{j} r\right)\right|} \int_{A\left(0,2^{j} r\right)} f(y) d y \\
& \leq C r^{\beta} \sum_{j=1}^{\infty}\left(2^{j} r\right)^{-\beta} \Phi_{\infty}^{-1}\left(\left(2^{j} r\right)^{-N}\right)\|f\|_{L^{\Phi}\left(A\left(0,2^{j} r\right)\right)}
\end{aligned}
$$




$$
\begin{aligned}
\leq & C r^{\beta}\left(\sum_{j=1}^{\infty}\left(\left(2^{j} r\right)^{\varepsilon-\beta} \omega\left(2^{j} r\right)^{-1} \Phi_{\infty}^{-1}\left(\left(2^{j} r\right)^{-N}\right)\right)^{q^{\prime}}\right)^{1 / q^{\prime}} \\
& \times\left(\sum_{j=1}^{\infty}\left(\left(2^{j} r\right)^{-\varepsilon} \omega\left(2^{j} r\right)\|f\|_{L^{\Phi}\left(A\left(0,2^{j} r\right)\right)}\right)^{q}\right)^{1 / q} .
\end{aligned}
$$

Here note from $\left(\Phi_{\infty} \omega 1 ; \beta\right)$ that

$$
\begin{aligned}
& \left(\sum_{j=1}^{\infty}\left(\left(2^{j} r\right)^{\varepsilon-\beta} \omega\left(2^{j} r\right)^{-1} \Phi_{\infty}^{-1}\left(\left(2^{j} r\right)^{-N}\right)\right)^{q^{\prime}}\right)^{1 / q^{\prime}} \\
& \quad \leq C r^{\varepsilon_{1}-\beta} \omega(r)^{-1} \Phi_{\infty}^{-1}\left(r^{-N}\right)\left(\sum_{j=1}^{\infty}\left(2^{j} r\right)^{\left(\varepsilon-\varepsilon_{1}\right) q^{\prime}}\right)^{1 / q^{\prime}} \\
& \quad \leq C r^{\varepsilon-\beta} \omega(r)^{-1} \Phi_{\infty}^{-1}\left(r^{-N}\right) .
\end{aligned}
$$

By (2), we have

$$
\begin{aligned}
& \left(\sum_{j=1}^{\infty}\left(\left(2^{j} r\right)^{-\varepsilon} \omega\left(2^{j} r\right)\|f\|_{L^{\Phi}\left(A\left(0,2^{j} r\right)\right)}\right)^{q}\right)^{1 / q} \\
& \quad \leq C\left(\sum_{j=1}^{\infty}\left(2^{j} r\right)^{-\varepsilon} \int_{(2 / 3) 2^{j} r}^{(4 / 3) 2^{j} r}\left(\omega(t)\|f\|_{L^{\Phi}(A(0, t))}\right)^{q} \frac{d t}{t}\right)^{1 / q} \\
& \quad \leq C\left(\sum_{j=1}^{\infty} \int_{2^{j-1} r}^{2^{j+1} r}\left(t^{-\varepsilon} \omega(t)\|f\|_{L^{\Phi}(A(0, t))}\right)^{q} \frac{d t}{t}\right)^{1 / q} \\
& \quad \leq C\left(\int_{r}^{\infty}\left(t^{-\varepsilon} \omega(t)\|f\|_{L^{\Phi}(A(0, t))}\right)^{q} \frac{d t}{t}\right)^{1 / q}
\end{aligned}
$$

Hence

$$
H_{\beta}^{\infty} f(r) \leq C r^{\varepsilon} \omega(r)^{-1} \Phi_{\infty}^{-1}\left(r^{-N}\right)\left(\int_{r}^{\infty}\left(t^{-\varepsilon} \omega(t)\|f\|_{L^{\Phi}(A(0, t))}\right)^{q} \frac{d t}{t}\right)^{1 / q} .
$$

For the case $0<q \leq 1$, by the fact that $(a+b)^{q} \leq a^{q}+b^{q}$ for all $a, b \geq 0$ instead of Hölder's inequality, we obtain the required inequality.

For a nonnegative function $f \in L_{l o c}^{1}\left(\mathbf{R}^{N}\right)$ and a real number $\beta$, set

$$
H_{\beta}^{0} f(r)=r^{\beta} \int_{B(0, r) \backslash B(0,1)}|y|^{-N-\beta} f(y) d y .
$$


Lemma 8. For a real number $\beta$, suppose that $\Phi_{\infty}(t)$ satisfies $\left(\Phi_{\infty} 2\right)$ and $\left(\Phi_{\infty} \omega 2 ; \beta\right) \quad t^{-\varepsilon_{2}-\beta} \omega(t)^{-1} \Phi_{\infty}^{-1}\left(t^{-N}\right)$ is almost increasing in $[1, \infty)$ for some $\varepsilon_{2}>0$.

If $0<\varepsilon<\varepsilon_{2}$, then there exists a constant $C>0$ such that

$$
H_{\beta}^{0} f(r) \leq C r^{-\varepsilon} \omega(r)^{-1} \Phi_{\infty}^{-1}\left(r^{-N}\right)\left(\int_{1 / 2}^{r}\left(t^{\varepsilon} \omega(t)\|f\|_{L^{\Phi}(A(0, t))}\right)^{q} \frac{d t}{t}\right)^{1 / q}
$$

for all $r \geq 1$ and nonnegative functions $f \in L_{\text {loc }}^{1}\left(\mathbf{R}^{N}\right)$.

Proof. We show only the case $1<q<\infty$ since the remaining case is easily treated. Let $f \in L_{l o c}^{1}\left(\mathbf{R}^{N}\right)$ be a nonnegative function on $\mathbf{R}^{N}$. Let $r \geq 1$ and $0<\varepsilon<\varepsilon_{2}$. Let $j_{0}$ be the largest integer such that $2^{-j_{0}+1} r \geq 1$. We have by Lemma 5 , Hölder's inequality, $\left(\Phi_{\infty} \omega 2 ; \beta\right)$ and (2)

$$
\begin{aligned}
& H_{\beta}^{0} f(r)=r^{\beta} \sum_{j=1}^{j_{0}} \int_{A\left(0,2^{-j} r\right) \backslash B(0,1)}|y|^{-N-\beta} f(y) d y \\
& \leq C r^{\beta} \sum_{j=1}^{j_{0}}\left(2^{-j} r\right)^{-\beta} \frac{1}{\left|A\left(0,2^{-j} r\right)\right|} \int_{A\left(0,2^{-j} r\right) \backslash B(0,1)} f(y) d y \\
& \leq C r^{\beta} \sum_{j=1}^{j_{0}}\left(2^{-j} r\right)^{-\beta} \Phi_{\infty}^{-1}\left(\left(2^{-j} r\right)^{-N}\right)\left\|f \chi_{\mathbf{R}^{N} \backslash B(0,1)}\right\|_{L^{\Phi}\left(A\left(0,2^{-j} r\right)\right)} \\
& \leq C r^{\beta}\left(\sum_{j=1}^{j_{0}}\left(\left(2^{-j} r\right)^{-\varepsilon-\beta} \omega\left(2^{-j} r\right)^{-1} \Phi_{\infty}^{-1}\left(\left(2^{-j} r\right)^{-N}\right)\right)^{q^{\prime}}\right)^{1 / q^{\prime}} \\
& \times\left(\sum_{j=1}^{j_{0}}\left(\left(2^{-j} r\right)^{\varepsilon} \omega\left(2^{-j} r\right)\left\|f \chi_{\mathbf{R}^{N} \backslash B(0,1)}\right\|_{L^{\Phi}\left(A\left(0,2^{-j} r\right)\right)}\right)^{q}\right)^{1 / q} \\
& \leq C r^{-\varepsilon_{2}} \omega(r)^{-1} \Phi_{\infty}^{-1}\left(r^{-N}\right)\left(\sum_{j=1}^{j_{0}}\left(2^{-j} r\right)^{\left(\varepsilon_{2}-\varepsilon\right) q^{\prime}}\right)^{1 / q^{\prime}} \\
& \times\left(\sum_{j=1}^{j_{0}}\left(\left(2^{-j} r\right)^{\varepsilon} \omega\left(2^{-j} r\right)\left\|f \chi_{\mathbf{R}^{N} \backslash B(0,1)}\right\|_{L^{\Phi}\left(A\left(0,2^{-j} r\right)\right)}\right)^{q}\right)^{1 / q} \\
& \leq C r^{-\varepsilon} \omega(r)^{-1} \Phi_{\infty}^{-1}\left(r^{-N}\right) \\
& \times\left(\sum_{j=1}^{j_{0}}\left(\left(2^{-j} r\right)^{\varepsilon} \omega\left(2^{-j} r\right)\left\|f \chi_{\mathbf{R}^{N} \backslash B(0,1)}\right\|_{L^{\Phi}\left(A\left(0,2^{-j} r\right)\right)}\right)^{q}\right)^{1 / q}
\end{aligned}
$$




$$
\begin{aligned}
& \leq C r^{-\varepsilon} \omega(r)^{-1} \Phi_{\infty}^{-1}\left(r^{-N}\right)\left(\int_{1 / 4}^{r}\left(t^{\varepsilon} \omega(t)\left\|f \chi_{\mathbf{R}^{N} \backslash B(0,1)}\right\|_{L^{\Phi}(A(0, t))}\right)^{q} \frac{d t}{t}\right)^{1 / q} \\
& \leq C r^{-\varepsilon} \omega(r)^{-1} \Phi_{\infty}^{-1}\left(r^{-N}\right)\left(\int_{1 / 2}^{r}\left(t^{\varepsilon} \omega(t)\|f\|_{L^{\Phi}(A(0, t))}\right)^{q} \frac{d t}{t}\right)^{1 / q}
\end{aligned}
$$

which gives the required result.

We present the boundedness of the maximal operator in $\mathscr{H}^{\Phi, q, \omega}\left(\mathbf{R}^{N}\right)$.

TheOREM 1. Suppose that $\Phi(x, t)$ satisfies $(\Phi 3 ; 0 ; p),(\Phi 3 ; \infty ; q),(\Phi 5 ; \eta)$ and $(\Phi 6 ; \tau)$ for $p>1, q>1, \eta>0$ and $\tau>0$ satisfying $\eta \leq q / N$ and $\tau \leq p$. Assume that $\Phi_{\infty}(t)$ satisfies $\left(\Phi_{\infty} 2\right),\left(\Phi_{\infty} \omega 1 ; 0\right)$ and $\left(\Phi_{\infty} \omega 2 ;-N\right)$. Then the maximal operator $M$ is bounded from $\mathscr{H}^{\Phi, q, \omega}\left(\mathbf{R}^{N}\right)$ to itself, that is,

$$
\|M f\|_{\mathscr{H}^{\Phi, q, \omega}\left(\mathbf{R}^{N}\right)} \leq C\|f\|_{\mathscr{H}^{\Phi, q, \omega}\left(\mathbf{R}^{N}\right)} \quad \text { for all } f \in \mathscr{H}^{\Phi, q, \omega}\left(\mathbf{R}^{N}\right) .
$$

REMARK 9. Let $\Phi(x, t)$ be as in Example 1 and let $\Phi_{\infty}(t)$ be as in Remark 8. If $\omega(r)=r^{v}$, then $\left(\Phi_{\infty} \omega 1 ; 0\right)$ and $\left(\Phi_{\infty} \omega 2 ;-N\right)$ hold when

$$
-N / p_{1}(\infty)<v<N\left(1-1 / p_{1}(\infty)\right) \text {. }
$$

Proof (Proof of Theorem 1). Let $f$ be a nonnegative measurable function on $\mathbf{R}^{N}$ such that $\|f\|_{\mathscr{H}^{\Phi, q, \omega}\left(\mathbf{R}^{N}\right)} \leq 1$. First we show

$$
\int_{2}^{\infty}\left(\omega(r)\|M f\|_{L^{\Phi}(A(0, r))}\right)^{q} \frac{d r}{r} \leq C .
$$

For $r \geq 2$, set

$$
\begin{aligned}
f & =f \chi_{B(0,1)}+f \chi_{B(0, r / 2) \backslash B(0,1)}+f \chi_{B(0,4 r) \backslash B(0, r / 2)}+f \chi_{\mathbf{R}^{N} \backslash B(0,4 r)} \\
& =f_{0}+f_{1, r}+f_{2, r}+f_{3, r} .
\end{aligned}
$$

For $f_{0}$, by Lemma 2 we have

$$
M f_{0}(x) \leq C|x|^{-N} \int_{B(0,1)} f(y) d y \leq C|x|^{-N}
$$

for $x \in \mathbf{R}^{N} \backslash B(0, r)$. By Lemmas 6 and 4

$$
\begin{aligned}
r^{N}\left\||\cdot|^{-N}\right\|_{L^{\Phi}\left(\mathbf{R}^{N} \backslash B(0, r)\right)} & \leq C\left\|M \chi_{B(0, r / 2)}\right\|_{L^{\Phi}\left(\mathbf{R}^{N} \backslash B(0, r)\right)} \\
& \leq C\left\|\chi_{B(0, r / 2)}\right\|_{L^{\Phi}\left(\mathbf{R}^{N}\right)} \leq C\left\{\Phi_{\infty}^{-1}\left(r^{-N}\right)\right\}^{-1} .
\end{aligned}
$$

Hence

$$
\left\|M f_{0}\right\|_{L^{\Phi}(A(0, r))} \leq C r^{-N}\left\{\Phi_{\infty}^{-1}\left(r^{-N}\right)\right\}^{-1} .
$$


Since $r^{\varepsilon_{2}-N} \omega(r)\left\{\Phi_{\infty}^{-1}\left(r^{-N}\right)\right\}^{-1}$ is almost decreasing in $[1, \infty)$ by $\left(\Phi_{\infty} \omega 2 ;-N\right)$, it follows that

$$
\begin{aligned}
\int_{2}^{\infty}\left(\omega(r)\left\|M f_{0}\right\|_{L^{\Phi}(A(0, r))}\right)^{q} \frac{d r}{r} & \leq C \int_{2}^{\infty}\left(r^{-N} \omega(r)\left\{\Phi_{\infty}^{-1}\left(r^{-N}\right)\right\}^{-1}\right)^{q} \frac{d r}{r} \\
& \leq C .
\end{aligned}
$$

For $f_{1, r}$, we find for $x \in \mathbf{R}^{N} \backslash B(0, r)$

$$
\begin{aligned}
M f_{1, r}(x) & \leq C|x|^{-N} \int_{B(0, r / 2) \backslash B(0,1)} f(y) d y \leq C(|x| / r)^{-N} H_{-N}^{0} f(r / 2) \\
& \leq C|x|^{-N} r^{N-\varepsilon_{2}^{\prime}} \omega(r)^{-1} \Phi_{\infty}^{-1}\left(r^{-N}\right)\left(\int_{1 / 2}^{r}\left(t^{\varepsilon_{2}^{\prime}} \omega(t)\|f\|_{L^{\Phi}(A(0, t))}\right)^{q} \frac{d t}{t}\right)^{1 / q}
\end{aligned}
$$

for $0<\varepsilon_{2}^{\prime}<\varepsilon_{2}$ by Lemma 8 . Hence, using (5), we have

$$
\begin{aligned}
& \int_{2}^{\infty}\left(\omega(r)\left\|M f_{1, r}\right\|_{L^{\Phi}(A(0, r))}\right)^{q} \frac{d r}{r} \\
& \quad \leq C \int_{2}^{\infty} r^{-\varepsilon_{2}^{\prime} q}\left\{\int_{1 / 2}^{r}\left(t^{\varepsilon_{2}^{\prime}} \omega(t)\|f\|_{L^{\Phi}(A(0, t))}\right)^{q} \frac{d t}{t}\right\} \frac{d r}{r} \\
& \leq C \int_{1 / 2}^{\infty}\left(t^{\varepsilon_{2}^{\prime}} \omega(t)\|f\|_{L^{\Phi}(A(0, t))}\right)^{q}\left(\int_{t}^{\infty} r^{-\varepsilon_{2}^{\prime} q} \frac{d r}{r}\right) \frac{d t}{t} \\
& \leq C \int_{1 / 2}^{\infty}\left(\omega(t)\|f\|_{L^{\Phi}(A(0, t))}\right)^{q} \frac{d t}{t} \leq C .
\end{aligned}
$$

For $f_{2, r}$, by Lemma 6

$$
\left\|M f_{2, r}\right\|_{L^{\Phi}(A(0, r))} \leq C\left\|f_{2, r}\right\|_{L^{\Phi}\left(\mathbf{R}^{N}\right)}=C\|f\|_{L^{\Phi}(B(0,4 r) \backslash B(0, r / 2))},
$$

which implies

$$
\int_{2}^{\infty}\left(\omega(r)\left\|M f_{2, r}\right\|_{L^{\Phi}(A(0, r))}\right)^{q} \frac{d r}{r} \leq C .
$$

For $f_{3, r}$, we find for $x \in B(0,2 r)$

$$
\begin{aligned}
M f_{3, r}(x) & \leq C \int_{\mathbf{R}^{N} \backslash B(0,4 r)} f(y)|y|^{-N} d y \leq C H_{0}^{\infty} f(2 r) \\
& \leq C r^{\varepsilon_{1}^{\prime}} \omega(r)^{-1} \Phi_{\infty}^{-1}\left(r^{-N}\right)\left(\int_{r}^{\infty}\left(t^{-\varepsilon_{1}^{\prime}} \omega(t)\|f\|_{L^{\Phi}(A(0, t))}\right)^{q} \frac{d t}{t}\right)^{1 / q}
\end{aligned}
$$


for $0<\varepsilon_{1}^{\prime}<\varepsilon_{1}$ by Lemma 7 . Hence, by Lemma 4

$$
\begin{aligned}
\left\|M f_{3, r}\right\|_{L^{\Phi}(A(0, r))} & \leq\left\|M f_{3, r}\right\|_{L^{\Phi}(B(0,2 r))} \\
& \leq C r^{\varepsilon_{1}^{\prime}} \omega(r)^{-1}\left(\int_{r}^{\infty}\left(t^{-\varepsilon_{1}^{\prime}} \omega(t)\|f\|_{L^{\Phi}(A(0, t))}\right)^{q} \frac{d t}{t}\right)^{1 / q}
\end{aligned}
$$

so that

$$
\begin{aligned}
& \int_{2}^{\infty}\left(\omega(r)\left\|M f_{3, r}\right\|_{L^{\Phi}(A(0, r))}\right)^{q} \frac{d r}{r} \\
& \quad \leq C \int_{2}^{\infty} r^{\varepsilon_{1}^{\prime} q}\left(\int_{r}^{\infty}\left(t^{-\varepsilon_{1}^{\prime}} \omega(t)\|f\|_{L^{\Phi}(A(0, t))}\right)^{q} \frac{d t}{t}\right) \frac{d r}{r} \\
& \quad \leq C \int_{2}^{\infty}\left(t^{-\varepsilon_{1}^{\prime}} \omega(t)\|f\|_{L^{\Phi}(A(0, t))}\right)^{q}\left(\int_{2}^{t} r^{\varepsilon_{1}^{\prime} q} \frac{d r}{r}\right) \frac{d t}{t} \\
& \quad \leq C \int_{2}^{\infty}\left(\omega(t)\|f\|_{L^{\Phi}(A(0, t))}\right)^{q} \frac{d t}{t} \leq C .
\end{aligned}
$$

Combining (6), (8), (9) and (11), we obtain (4). Finally we show

$$
\|M f\|_{L^{\Phi}(B(0,4))} \leq C
$$

since

$$
\|M f\|_{L^{\Phi}(B(0,2))}+\left(\int_{1}^{2}\left(\omega(r)\|M f\|_{L^{\Phi}(A(0, r))}\right)^{q} \frac{d r}{r}\right)^{1 / q} \leq C\|M f\|_{L^{\Phi}(B(0,4))} .
$$

Set

$$
f=f \chi_{B(0,8)}+f \chi_{\mathbf{R}^{N} \backslash B(0,8)}=f_{4}+f_{5} .
$$

By Lemmas 6 and 2,

$$
\left\|M f_{4}\right\|_{L^{\Phi}(B(0,4))} \leq C\|f\|_{L^{\Phi}(B(0,8))} \leq C
$$

and

$$
\begin{aligned}
\left\|M f_{5}\right\|_{L^{\Phi}(B(0,4))} & =\left\|M f_{3,2}\right\|_{L^{\Phi}(B(0,4))} \\
& \leq C\left(\int_{2}^{\infty}\left(\omega(t)\|f\|_{L^{\Phi}(A(0, t))}\right)^{q} \frac{d t}{t}\right)^{1 / q} \leq C
\end{aligned}
$$

by (10). 


\section{Boundedness of the Hardy operator}

For a locally integrable function $f$ on $\mathbf{R}^{N}$ and $\beta \in \mathbf{R}$, the Hardy functions $\hat{H}_{\beta}^{\infty} f$ and $\hat{H}_{\beta}^{0} f$ are defined by

$$
\hat{H}_{\beta}^{\infty} f(x)=|x|^{\beta} \int_{\mathbf{R}^{N} \backslash(B(0,|x|) \cup B(0,1))}|y|^{-N-\beta}|f(y)| d y
$$

and

$$
\hat{H}_{\beta}^{0} f(x)=|x|^{\beta} \int_{B(0,|x|) \backslash B(0,1)}|y|^{-N-\beta}|f(y)| d y,
$$

respectively.

LeMMA 9. For a real number $\beta$, suppose that $\Phi(x, t)$ satisfies $(\Phi 3 ; \infty ; q)$ and $(\Phi 5 ; \eta)$ for $q \geq 1$ and $\eta>0$ satisfying $\eta \leq q / N$. Assume that $(\Phi \omega ; \beta) \quad t^{-\varepsilon_{3}+\beta}\left\{\bar{\Phi}^{-1}\left(0, t^{-N}\right)\right\}^{-1}$ is almost increasing in $(0,1]$ for some $\varepsilon_{3}>0$. Then there exists a constant $C>0$ such that

$$
\left\||\cdot|^{\beta}\right\|_{L^{\Phi}(B(0, r))} \leq C r^{\beta}\left\{\bar{\Phi}^{-1}\left(0, r^{-N}\right)\right\}^{-1}
$$

for all $0<r \leq 1$.

Proof. Let $0<r \leq 1$. First note from $(1),(\Phi 2)$ and $(\Phi 3 ; \infty ; q)$ that

$$
r^{-N}<|x|^{-N} \leq 2 A_{1} A_{2, \infty, q} A_{3} \bar{\Phi}\left(0,|x|^{-N / q}\right)
$$

for $x \in B(0, r)$, so that we have by Lemma 3 (2) and (4)

$$
|x| \leq C\left\{\bar{\Phi}^{-1}\left(0, r^{-N}\right)\right\}^{-q / N}
$$

for $x \in B(0, r)$ and

$$
\bar{\Phi}^{-1}\left(0, r^{-N}\right) \geq \bar{\Phi}^{-1}(0,1)>0 .
$$

Therefore we find by $(\Phi 5 ; \eta)$ and $\eta \leq q / N$

$$
\int_{B(0, r)} \bar{\Phi}\left(x, \bar{\Phi}^{-1}\left(0, r^{-N}\right)\right) d x \leq C \int_{B(0, r)} \bar{\Phi}\left(0, \bar{\Phi}^{-1}\left(0, r^{-N}\right)\right) d x \leq C,
$$

so that

$$
\left\|\chi_{B(0, r)}\right\|_{L^{\Phi}\left(\mathbf{R}^{N}\right)} \leq C\left\{\bar{\Phi}^{-1}\left(0, r^{-N}\right)\right\}^{-1} .
$$

Hence we have by $(\Phi \omega ; \beta)$ 


$$
\begin{aligned}
\left\||\cdot|^{\beta}\right\|_{L^{\Phi}(B(0, r))} & \leq \sum_{j=1}^{\infty}\left\||\cdot|^{\beta}\right\|_{L^{\Phi}\left(A\left(0,2^{-j} r\right)\right)} \\
& \leq C \sum_{j=1}^{\infty}\left(2^{-j} r\right)^{\beta}\left\{\bar{\Phi}^{-1}\left(0,\left(2^{-j} r\right)^{-N}\right)\right\}^{-1} \\
& \leq C r^{-\varepsilon_{3}+\beta}\left\{\bar{\Phi}^{-1}\left(0, r^{-N}\right)\right\}^{-1} \sum_{j=1}^{\infty}\left(2^{-j} r\right)^{\varepsilon_{3}} \\
& \leq C r^{\beta}\left\{\bar{\Phi}^{-1}\left(0, r^{-N}\right)\right\}^{-1},
\end{aligned}
$$

as required.

THEOREM 2. For a real number $\beta$, suppose that $\Phi(x, t)$ satisfies $(\Phi 3 ; \infty ; q)$ and $(\Phi 5 ; \eta)$ for $q \geq 1$ and $\eta>0$ satisfying $\eta \leq q / N$. Assume that $\Phi(x, t)$ satisfies $(\Phi \omega ; \beta)$ and $\Phi_{\infty}(t)$ satisfies $\left(\Phi_{\infty} 2\right)$ and $\left(\Phi_{\infty} \omega 1 ; \beta\right)$. Then there exists a constant $C>0$ such that

$$
\left\|\hat{H}_{\beta}^{\infty} f\right\|_{\mathscr{H}^{\Phi, q, \omega}\left(\mathbf{R}^{N}\right)} \leq C\|f\|_{\mathscr{H}^{\Phi, q, \omega}\left(\mathbf{R}^{N}\right)}
$$

for all $f \in \mathscr{H}^{\Phi, q, \omega}\left(\mathbf{R}^{N}\right)$.

Proof. Let $f$ be a nonnegative measurable function on $\mathbf{R}^{N}$ such that $\|f\|_{\mathscr{H}^{\Phi, q, \omega}\left(\mathbf{R}^{N}\right)} \leq 1$. Let $r \geq 2$. Then we have by Lemmas 4 and 7

$$
\begin{aligned}
\left\|\hat{H}_{\beta}^{\infty} f\right\|_{L^{\Phi}(A(0, r))} & \leq C H_{\beta}^{\infty} f(r / 2)\|1\|_{L^{\Phi}(A(0, r))} \\
& \leq C r^{\varepsilon_{1}^{\prime}} \omega(r)^{-1}\left(\int_{r / 2}^{\infty}\left(t^{-\varepsilon_{1}^{\prime}} \omega(t)\|f\|_{L^{\Phi}(A(0, t))}\right)^{q} \frac{d t}{t}\right)^{1 / q}
\end{aligned}
$$

for $0<\varepsilon_{1}^{\prime}<\varepsilon_{1}$. Therefore, as in the proof of Theorem 1, we obtain

$$
\int_{2}^{\infty}\left(\omega(r)\left\|\hat{H}_{\beta}^{\infty} f\right\|_{L^{\Phi}(A(0, r))}\right)^{q} \frac{d r}{r} \leq C .
$$

Finally we show

$$
\left\|\hat{H}_{\beta}^{\infty} f\right\|_{L^{\Phi}(B(0,4))} \leq C .
$$

Note from Lemmas 7 and 2 that

$$
\begin{aligned}
\hat{H}_{\beta}^{\infty} f(x) & \leq 2^{\beta}|x|^{\beta} H_{\beta}^{\infty} f(1 / 2) \\
& \leq C|x|^{\beta}\left\{\left(\int_{1}^{\infty}\left(\omega(t)\|f\|_{L^{\Phi}(A(0, t))}\right)^{q} \frac{d t}{t}\right)^{1 / q}+\int_{B(0,2) \backslash B(0,1)} f(y) d y\right\} \\
& \leq C|x|^{\beta} .
\end{aligned}
$$


Hence we obtain by Lemma 9

$$
\left\|\hat{H}_{\beta}^{\infty} f\right\|_{L^{\Phi}(B(0,4))} \leq C\left\||\cdot|^{\beta}\right\|_{L^{\Phi}(B(0,4))} \leq C,
$$

as required.

In the same manner, using Lemma 8 instead of Lemma 7, we can prove the following result.

THEOREM 3. For a real number $\beta$, suppose that $\Phi(x, t)$ satisfies $(\Phi 3 ; \infty ; q)$ and $(\Phi 5 ; \eta)$ for $q \geq 1$ and $\eta>0$ satisfying $\eta \leq q / N$. Assume that $\Phi(x, t)$ satisfies $(\Phi \omega ; \beta)$ and $\Phi_{\infty}(t)$ satisfies $\left(\Phi_{\infty} 2\right)$ and $\left(\Phi_{\infty} \omega 2 ; \beta\right)$. Then there exists a constant $C>0$ such that

$$
\left\|\hat{H}_{\beta}^{0} f\right\|_{\mathscr{H}^{\Phi, q, \omega}\left(\mathbf{R}^{N}\right)} \leq C\|f\|_{\mathscr{H}^{\Phi, q, \omega}\left(\mathbf{R}^{N}\right)}
$$

for all $f \in \mathscr{H}^{\Phi, q, \omega}\left(\mathbf{R}^{N}\right)$.

In fact, note that

$$
\hat{H}_{\beta}^{0} f(x) \leq C|x|^{\beta} \int_{B(0,4) \backslash B(0,1)}|f(y)| d y \leq C|x|^{\beta}
$$

for $x \in B(0,4)$ and $f \in \mathscr{H}^{\Phi, q, \omega}\left(\mathbf{R}^{N}\right)$ with $\|f\|_{\mathscr{H}^{\Phi, q, \omega}\left(\mathbf{R}^{N}\right)} \leq 1$.

\section{Sobolev's inequality}

For $0<\alpha<N$, the Riesz potential $I_{\alpha} f$ is defined by

$$
I_{\alpha} f(x)=\int_{\mathbf{R}^{N}}|x-y|^{\alpha-N} f(y) d y
$$

for a locally integrable function $f$ on $\mathbf{R}^{N}$.

Lemma 10 . Assume that $\Phi_{\infty}(t)$ satisfies $\left(\Phi_{\infty} 2\right)$ and $\left(\Phi_{\infty} \omega 1 ;-\alpha\right)$ for $\varepsilon_{1}>0$. Then, for $0<\varepsilon<\varepsilon_{1}$, there exists a constant $C>0$ such that, for all $x \in B(0,2 r)$ with $r \geq 1$ and nonnegative functions $f \in L_{l o c}^{1}\left(\mathbf{R}^{N}\right)$,

$$
\begin{aligned}
& \left|I_{\alpha}\left(f \chi_{\mathbf{R}^{N} \backslash B(0,4 r)}\right)(x)\right| \\
& \quad \leq C r^{\varepsilon+\alpha} \omega(r)^{-1} \Phi_{\infty}^{-1}\left(r^{-N}\right)\left(\int_{r}^{\infty}\left(t^{-\varepsilon} \omega(t)\|f\|_{L^{\Phi}(A(0, t))}\right)^{q} \frac{d t}{t}\right)^{1 / q} .
\end{aligned}
$$

Proof. Let $f \in L_{l o c}^{1}\left(\mathbf{R}^{N}\right)$ be a nonnegative function on $\mathbf{R}^{N}$. Let $r \geq 1$, $x \in B(0,2 r)$ and $0<\varepsilon<\varepsilon_{1}$. Note from Lemma 7 with $\beta=-\alpha$ that 


$$
\begin{aligned}
& \left|I_{\alpha}\left(f \chi_{\mathbf{R}^{N} \backslash B(0,4 r)}\right)(x)\right| \\
& \quad \leq C \int_{\mathbf{R}^{N} \backslash B(0,4 r)}|y|^{\alpha-N} f(y) d y \\
& \quad \leq C r^{\varepsilon+\alpha} \omega(r)^{-1} \Phi_{\infty}^{-1}\left(r^{-N}\right)\left(\int_{r}^{\infty}\left(t^{-\varepsilon} \omega(t)\|f\|_{L^{\Phi}(A(0, t))}\right)^{q} \frac{d t}{t}\right)^{1 / q},
\end{aligned}
$$

as required.

Lemma 11. Assume that $\Phi_{\infty}(t)$ satisfies $\left(\Phi_{\infty} 2\right)$ and $\left(\Phi_{\infty} \omega 2 ;-N\right)$ for $\varepsilon_{2}>0$. Then, for $0<\varepsilon<\varepsilon_{2}$, there exists a constant $C>0$ such that for all $x \in \mathbf{R}^{N} \backslash B(0, r)$ with $r \geq 1$ and nonnegative functions $f \in L_{\text {loc }}^{1}\left(\mathbf{R}^{N}\right)$,

$$
\begin{aligned}
\left|I_{\alpha}\left(f \chi_{B(0, r / 2) \backslash B(0,1)}\right)(x)\right| \leq & C(|x| / r)^{\alpha-N} r^{-\varepsilon+\alpha} \omega(r)^{-1} \Phi_{\infty}^{-1}\left(r^{-N}\right) \\
& \times\left(\int_{1 / 2}^{r}\left(t^{\varepsilon} \omega(t)\|f\|_{L^{\Phi}(A(0, t))}\right)^{q} \frac{d t}{t}\right)^{1 / q} .
\end{aligned}
$$

Proof. Let $f \in L_{\text {loc }}^{1}\left(\mathbf{R}^{N}\right)$ be a nonnegative function on $\mathbf{R}^{N}$. Let $r \geq 1$, $x \in \mathbf{R}^{N} \backslash B(0, r)$ and $0<\varepsilon<\varepsilon_{2}$. Note that

$$
\begin{aligned}
\left|I_{\alpha}\left(f \chi_{B(0, r / 2) \backslash B(0,1)}\right)(x)\right| & \leq C|x|^{\alpha-N} \int_{B(0, r / 2) \backslash B(0,1)} f(y) d y \\
& =C(|x| / r)^{\alpha-N} r^{\alpha} H_{-N}^{0} f(r / 2),
\end{aligned}
$$

so that Lemma 8 with $\beta=-N$ gives the required result.

We consider a function

$$
\Psi(x, t)=t \psi(x, t): \mathbf{R}^{N} \times[0, \infty) \rightarrow[0, \infty)
$$

satisfying the conditions $(\Phi 1)-(\Phi 4)$ with $\phi$ replaced by $\psi$.

Now we consider the following conditions:

$\left(\Phi_{\infty} 2^{\prime}\right)$ there exists a constant $Q \geq 1$ such that

$$
\Phi_{\infty}\left(g^{*}(x)\right) \leq Q(1+|x|)^{-N}
$$

for all $x \in \mathbf{R}^{N}$, where $g^{*}(x)=\max \{g(x), M g(x)\}$;

$(\Phi \alpha) \quad r \mapsto r^{\varepsilon+\alpha} \bar{\Phi}^{-1}\left(x, r^{-N}\right)$ is uniformly almost decreasing on $(0, \infty)$ for some $\varepsilon>0$;

$(\Psi \Phi \alpha) \quad$ there exists a constant $Q \geq 1$ such that

$$
\Psi\left(x, t \Phi(x, t)^{-\alpha / N}\right) \leq Q \Phi(x, t)
$$

for all $x \in \mathbf{R}^{N}$ and $t>0$. 
REMARK 10. Let $\Phi(x, t)$ be as in Example 1 and let $\Phi_{\infty}(t)$ be as in Remark 8 with $\tau=1$. Assume

$$
\inf _{x \in \mathbf{R}^{N}}\left(N-\alpha p_{1}(x)\right)>0 \quad \text { and } \quad \inf _{x \in \mathbf{R}^{N}}\left(N-\alpha p_{2}(x)\right)>0 .
$$

Then $\Phi(x, t)$ satisfies $(\Phi \alpha)$ and $\Phi_{\infty}(t)$ satisfies $\left(\Phi_{\infty} 2^{\prime}\right)$.

Set

$$
\Psi(x, t)= \begin{cases}t^{p_{1}^{*}(x)} \prod_{j=1}^{k_{1}}\left(L_{e-1}^{(j)}(1 / t)\right)^{-q_{1, j}(x) p_{1}^{*}(x) / p_{1}(x)} & \text { if } 0 \leq t \leq 1 \\ t^{p_{2}^{*}(x)} \prod_{j=1}^{k_{2}}\left(L_{e-1}^{(j)}(t)\right)^{q_{2, j}(x) p_{2}^{*}(x) / p_{2}(x)} & \text { if } t \geq 1,\end{cases}
$$

where

$$
\frac{1}{p_{i}^{*}(x)}=\frac{1}{p_{i}(x)}-\frac{\alpha}{N}
$$

for $i=1,2$. Then $\Psi(x, t)$ satisfies $(\Psi \Phi \alpha)$.

As Sobolev's inequality for Riesz potentials of functions in Musielak-Orlicz spaces $L^{\Phi}\left(\mathbf{R}^{N}\right)$, we give the following lemma ([30, Corollary 6.5]). Here we shall state our result without assumptions

$\left(\Phi_{\infty} 3\right) \quad r \mapsto r^{\gamma} \Phi_{\infty}^{-1}\left(r^{-N}\right)$ is almost increasing on $[1, \infty)$ for some $0<\gamma<$ $N$ and

$\left(\Phi_{\infty} \alpha\right) \quad r \mapsto r^{\varepsilon+\alpha} \Phi_{\infty}^{-1}\left(r^{-N}\right)$ is almost decreasing on $[1, \infty)$ for some $\varepsilon>0$, which are assumed in [30] (see Remark 11 below).

Lemma 12. Suppose $\Phi(x, t)$ satisfies $(\Phi 3 ; 0 ; p),(\Phi 3 ; \infty ; q),(\Phi 5 ; \eta),(\Phi 6 ; \tau)$ and $(\Phi \alpha)$ for $p>1, q>1, \eta>0$ and $\tau>0$ satisfying $\eta \leq q / N$ and $\tau \leq p$. For the function $\Phi_{\infty}(t)$, assume $\left(\Phi_{\infty} 2^{\prime}\right)$ holds. Further, assume that $\Psi(x, t)$ satisfies $(\Psi \Phi \alpha)$. Then there exists a constant $C>0$ such that

$$
\left\|I_{\alpha} f\right\|_{L^{\Psi}\left(\mathbf{R}^{N}\right)} \leq C\|f\|_{L^{\Phi}\left(\mathbf{R}^{N}\right)}
$$

for all $f \in L^{\Phi}\left(\mathbf{R}^{N}\right)$.

Remark 11. Assumptions $(\Phi 3 ; 0 ; p)$ and $(\Phi 3 ; \infty ; q)$ imply $\left(\Phi_{\infty} 3\right)$ and $(\Phi \alpha)$ implies $\left(\Phi_{\infty} \alpha\right)$.

In fact, we see from $(\Phi 3 ; 0 ; p)$ and $(\Phi 3 ; \infty ; q)$ that there exist constants $c>0$ and $\varepsilon>0$ such that

$$
t^{-(1+\varepsilon)} \Phi(x, t) \leq c s^{-(1+\varepsilon)} \Phi(x, s)
$$

for all $0<t<s$, so that for $0<t<s \leq \max \left\{1, \Phi_{\infty}^{-1}(1)\right\}$, there exists a point $x_{0} \in \mathbf{R}^{N}$ such that $g\left(x_{0}\right) \leq t<s \leq \max \left\{1, \Phi_{\infty}^{-1}(1)\right\}$ and

$$
t^{-(1+\varepsilon)} \Phi_{\infty}(t) \leq Q t^{-(1+\varepsilon)} \Phi\left(x_{0}, t\right) \leq c Q s^{-(1+\varepsilon)} \Phi\left(x_{0}, s\right) \leq c Q^{2} s^{-(1+\varepsilon)} \Phi_{\infty}(s)
$$


by $\left(\Phi_{\infty} 1\right)$ and $g \in L^{\tau}\left(\mathbf{R}^{N}\right)$, where $Q$ is the constant appearing in $\left(\Phi_{\infty} 1\right)$. Since $\Phi_{\infty}^{-1}\left(r^{-N}\right) \leq \Phi_{\infty}^{-1}(1)$ for all $r \geq 1$, we see that $\left(\Phi_{\infty} 3\right)$ holds with $\gamma=N /(1+\varepsilon)$. Similarly we can show that $(\Phi \alpha)$ implies $\left(\Phi_{\infty} \alpha\right)$.

Further, as the definition of $\Phi_{\infty}(t)$, we consider a convex function $\Psi_{\infty}(t)=$ $t \psi_{\infty}(t):[0, \infty) \rightarrow[0, \infty)$ such that $\psi_{\infty}(t)>0$ for $t>0, \psi_{\infty}(t)$ is increasing on $[0, \infty)$ and satisfies the doubling condition and

$\left(\Psi_{\infty} 1\right)$ there exists a constant $Q \geq 1$ such that

$$
Q^{-1} \Psi(x, t) \leq \Psi_{\infty}(t) \leq Q \Psi(x, t) \quad \text { whenever } g(x) \leq t \leq 1,
$$

where $g$ is the function appearing in $(\Phi 6 ; \tau)$.

Now we show the Sobolev type inequality for Riesz potentials of functions in $\mathscr{H}^{\Phi, q, \omega}\left(\mathbf{R}^{N}\right)$.

TheOREM 4. Suppose $\Phi(x, t)$ satisfies $(\Phi 3 ; 0 ; p),(\Phi 3 ; \infty ; q),(\Phi 5 ; \eta),(\Phi 6 ; \tau)$ and $(\Phi \alpha)$ for $p>1, q>1, \eta>0$ and $\tau>0$ satisfying $\eta \leq q / N$ and $\tau \leq p$. Assume that $\Psi(x, t)$ satisfies $(\Psi \Phi \alpha)$. For the function $\Phi_{\infty}(t)$, assume $\left(\Phi_{\infty} 2^{\prime}\right)$, $\left(\Phi_{\infty} \omega 1 ;-\alpha\right)$ and $\left(\Phi_{\infty} \omega 2 ;-N\right)$ hold. Then there exists a constant $C>0$ such that

$$
\left\|I_{\alpha} f\right\|_{\mathscr{H}^{\Psi, q, \omega}\left(\mathbf{R}^{N}\right)} \leq C\|f\|_{\mathscr{H}^{\Phi, q, \omega}\left(\mathbf{R}^{N}\right)}
$$

for all $f \in \mathscr{H}^{\Phi, q, \omega}\left(\mathbf{R}^{N}\right)$.

REMARK 12. Suppose $\Phi(x, t)$ satisfies $(\Phi \alpha)$ and $\Psi(x, t)$ satisfies $(\Psi \Phi \alpha)$. For the function $\Phi_{\infty}(t)$, assume $\left(\Phi_{\infty} 2^{\prime}\right)$ holds.

To show Theorem 4 , we need to verify that the following conditions hold: $\left(\Psi_{\infty} 2\right) \quad$ there exists a constant $Q \geq 1$ such that

$$
\Psi_{\infty}(g(x)) \leq Q(1+|x|)^{-N}
$$

for all $x \in \mathbf{R}^{N}$;

$\left(\Psi_{\infty} \Phi_{\infty} \alpha\right)$ there exists a constant $Q>0$ such that

$$
\sup _{t \geq 1} t^{\alpha} \Phi_{\infty}^{-1}\left(t^{-N}\right)\left\{\Psi_{\infty}^{-1}\left(t^{-N}\right)\right\}^{-1} \leq Q
$$

First we show that $\left(\Psi_{\infty} 2\right)$ holds. Since we have by $(\Psi \Phi \alpha)$ and $\left(\Psi_{\infty} 1\right)$

$$
\begin{aligned}
\Phi(x, g(x)) & \geq C \Psi\left(x, g(x) \Phi(x, g(x))^{-\alpha / N}\right) \\
& \geq C \Psi\left(x, g(x) \Phi(x, 1)^{-\alpha / N}\right) \geq C \Psi(x, g(x)) \geq C \Psi_{\infty}(g(x)),
\end{aligned}
$$

we find by $\left(\Phi_{\infty} 1\right)$ and $\left(\Phi_{\infty} 2\right)$

$$
\Psi_{\infty}(g(x)) \leq C \Phi(x, g(x)) \leq C \Phi_{\infty}(g(x)) \leq C(1+|x|)^{-N} .
$$


Next we show that $\left(\Psi_{\infty} \Phi_{\infty} \alpha\right)$ holds. For $0<t \leq \max \left\{1, \Phi_{\infty}^{-1}(1)\right\}$, there exists a point $x_{0} \in \mathbf{R}^{N}$ such that $g\left(x_{0}\right) \leq t \leq \max \left\{1, \Phi_{\infty}^{-1}(1)\right\}$ by $g \in L^{\tau}\left(\mathbf{R}^{N}\right)$. Then note that

$$
t \Phi\left(x_{0}, t\right)^{-\alpha / N} \geq C t \geq C g\left(x_{0}\right)
$$

and $t \Phi\left(x_{0}, t\right)^{-\alpha / N} \leq C$ by $(\Phi \alpha)$, so that we find by $\left(\Phi_{\infty} 1\right)$ and $\left(\Psi_{\infty} 1\right)$

$$
\Psi_{\infty}\left(t \Phi_{\infty}(t)^{-\alpha / N}\right) \leq C \Phi_{\infty}(t) .
$$

Since $\Phi_{\infty}^{-1}\left(r^{-N}\right) \leq \Phi_{\infty}^{-1}(1)$ for all $r \geq 1$, we obtain that $\left(\Psi_{\infty} \Phi_{\infty} \alpha\right)$ holds.

Proof (Proof of Theorem 4). Let $f$ be a nonnegative measurable function on $\mathbf{R}^{N}$ such that $\|f\|_{\mathscr{H}^{\Phi, q, \omega}\left(\mathbf{R}^{N}\right)} \leq 1$. For $r \geq 2$ set

$$
\begin{aligned}
f & =f \chi_{B(0,1)}+f \chi_{B(0, r / 2) \backslash B(0,1)}+f \chi_{B(0,4 r) \backslash B(0, r / 2)}+f \chi_{\mathbf{R}^{N} \backslash B(0,4 r)} \\
& =f_{0}+f_{1, r}+f_{2, r}+f_{3, r} .
\end{aligned}
$$

For $f_{0}$, we see that

$$
I_{\alpha} f_{0}(x) \leq C|x|^{\alpha-N} \int_{B(0,1)} f(y) d y \leq C|x|^{\alpha-N}
$$

for $x \in \mathbf{R}^{N} \backslash B(0, r)$ by Lemma 2. By Lemmas 12 and 4

$$
\begin{aligned}
r^{N}\left\||\cdot|^{\alpha-N}\right\|_{L^{\Psi}\left(\mathbf{R}^{N} \backslash B(0, r)\right)} & \\
& \leq C\left\|I_{\alpha} \chi_{B(0, r / 2)}\right\|_{L^{\Psi}\left(\mathbf{R}^{N} \backslash B(0, r)\right)} \\
& \leq C\left\|\chi_{B(0, r / 2)}\right\|_{L^{\Phi}\left(\mathbf{R}^{N}\right)} \leq C\left\{\Phi_{\infty}^{-1}\left(r^{-N}\right)\right\}^{-1} .
\end{aligned}
$$

Hence

$$
\left\|I_{\alpha} f_{0}\right\|_{L^{\Psi}(A(0, r))} \leq C r^{-N}\left\{\Phi_{\infty}^{-1}\left(r^{-N}\right)\right\}^{-1},
$$

and using $\left(\Phi_{\infty} \omega 2 ;-N\right)$, we have (cf. (6))

$$
\int_{2}^{\infty}\left(\omega(r)\left\|I_{\alpha} f_{0}\right\|_{L^{\Psi}(A(0, r))}\right)^{q} \frac{d r}{r} \leq C .
$$

For $f_{1, r}$, by Lemma 11 and (12), we have

$$
\left\|I_{\alpha} f_{1, r}\right\|_{L^{\Psi}(A(0, r))} \leq C r^{-\varepsilon_{2}^{\prime}} \omega(r)^{-1}\left(\int_{1 / 2}^{r}\left(t^{\varepsilon_{2}^{\prime}} \omega(t)\|f\|_{L^{\Phi}(A(0, t))}\right)^{q} \frac{d t}{t}\right)^{1 / q}
$$

for $0<\varepsilon_{2}^{\prime}<\varepsilon_{2}$, which implies (cf. (8))

$$
\int_{2}^{\infty}\left(\omega(r)\left\|I_{\alpha} f_{1, r}\right\|_{L^{\Psi}(A(0, r))}\right)^{q} \frac{d r}{r} \leq C .
$$


For $f_{2, r}$, we use Lemma 12 and have

$$
\left\|I_{\alpha} f_{2, r}\right\|_{L^{\Psi}(A(0, r))} \leq C\|f\|_{L^{\Phi}(B(0,4 r) \backslash B(0, r / 2))} .
$$

Hence

$$
\int_{2}^{\infty}\left(\omega(r)\left\|I_{\alpha} f_{2, r}\right\|_{L^{\Psi}(A(0, r))}\right)^{q} \frac{d r}{r} \leq C .
$$

To treat $f_{3, r}$, we remark that Lemma 4 holds for $\Psi$ by $\left(\Psi_{\infty} 2\right)$ and hence

$$
\|1\|_{L^{\Psi}(B(0, r))} \leq C\left\{\Psi_{\infty}^{-1}\left(r^{-N}\right)\right\}^{-1} \leq C r^{-\alpha}\left\{\Phi_{\infty}^{-1}\left(r^{-N}\right)\right\}^{-1}
$$

by $\left(\Psi_{\infty} \Phi_{\infty} \alpha\right)$. Thus, we find by Lemma 10

$$
\begin{aligned}
& \left\|I_{\alpha} f_{3, r}\right\|_{L^{\Psi}(A(0, r))} \\
& \quad \leq\left\|I_{\alpha} f_{3, r}\right\|_{L^{\Psi}(B(0, r))} \\
& \quad \leq \operatorname{Cr}^{\varepsilon_{1}^{\prime}} \omega(r)^{-1}\left(\int_{r}^{\infty}\left(t^{-\varepsilon_{1}^{\prime}} \omega(t)\|f\|_{L^{\Phi}(A(0, t))}\right)^{q} \frac{d t}{t}\right)^{1 / q}
\end{aligned}
$$

for $0<\varepsilon_{1}^{\prime}<\varepsilon_{1}$. It then follows that

$$
\int_{2}^{\infty}\left(\omega(r)\left\|I_{\alpha} f_{3, r}\right\|_{L^{\Psi}(A(0, r))}\right)^{q} \frac{d r}{r} \leq C .
$$

Combining (13), (14), (15) and (17), we obtain

$$
\int_{2}^{\infty}\left(\omega(r)\left\|I_{\alpha} f\right\|_{L^{\Psi}(A(0, r))}\right)^{q} \frac{d r}{r} \leq C .
$$

Finally, Lemma 12 and (16) with $r=2$ yield

$$
\begin{aligned}
\left\|I_{\alpha} f\right\|_{L^{\Psi}(B(0,2))}+\left(\int_{1}^{2}\left(\omega(r)\left\|I_{\alpha} f\right\|_{L^{\Psi}(A(0, r))}\right)^{q} \frac{d r}{r}\right)^{1 / q} \\
\quad \leq C\left\|I_{\alpha} f\right\|_{L^{\Psi}(B(0,4))} \\
\quad \leq C\left(\|f\|_{L^{\Phi}(B(0,8))}+\int_{1}^{\infty}\left(\omega(t)\|f\|_{L^{\Phi}(A(0, t))}\right)^{q} \frac{d t}{t}\right) \leq C,
\end{aligned}
$$

which proves the theorem.

REMARK 13. Let $\Phi$ be as in Example 1 and let $\Phi_{\infty}(t)$ be as in Remark 8 . If $\omega(r)=r^{v}$, then $\left(\Phi_{\infty} \omega 1 ;-\alpha\right)$ and $\left(\Phi_{\infty} \omega 2 ;-N\right)$ hold when

$$
\alpha-N / p_{1}(\infty)<v<N\left(1-1 / p_{1}(\infty)\right) .
$$


REMARK 14. Let $\Phi(x, t), \Phi_{\infty}(t)$ and $\Psi(x, t)$ be as in Example 1, Remark 8 and Remark 10. Assume

$$
\inf _{x \in \mathbf{R}^{N}}\left(N-\alpha p_{1}(x)\right)>0 .
$$

Set $\bar{\psi}_{\infty}(t)=\sup _{0 \leq s \leq t}\left\{s^{p_{1}^{*}(\infty)-1} \prod_{j=1}^{k_{1}}\left(L_{e-1}^{(j)}(1 / s)\right)^{-q_{1, j}(\infty) p_{1}^{*}(\infty) / p_{1}(\infty)}\right\}$ and

$$
\Psi_{\infty}(t)=\int_{0}^{t} \bar{\psi}_{\infty}(r) \frac{d r}{r}
$$

where

$$
\frac{1}{p_{1}^{*}(\infty)}=\frac{1}{p_{1}(\infty)}-\frac{\alpha}{N}
$$

Then $\Psi_{\infty}(t)$ satisfies $\left(\Psi_{\infty} 1\right)$.

\section{Sobolev's inequality for the generalized Riesz potential}

To obtain general results, for $0<\alpha<N$ and an integer $k \geq 1$, we define the generalized Riesz potential $I_{\alpha, k} f$ of order $\alpha$ of a locally integrable function $f$ on $\mathbf{R}^{N}$ by

$$
\begin{aligned}
I_{\alpha, k} f(x)= & \int_{B(0,1)} I_{\alpha}(x-y) f(y) d y \\
& +\int_{\mathbf{R}^{N} \backslash B(0,1)}\left\{I_{\alpha}(x-y)-\sum_{\{\mu:|\mu| \leq k-1\}} \frac{x^{\mu}}{\mu !}\left(D^{\mu} I_{\alpha}\right)(-y)\right\} f(y) d y,
\end{aligned}
$$

where $I_{\alpha}(x)=|x|^{\alpha-N}($ see $[32,33])$.

Set

$$
\tilde{I}_{\alpha, k}(x, y)=I_{\alpha}(x-y)-\sum_{\{\mu:|\mu| \leq k-1\}} \frac{x^{\mu}}{\mu !}\left(D^{\mu} I_{\alpha}\right)(-y)
$$

and

$$
\tilde{I}_{\alpha, k} f(x)=\int_{\mathbf{R}^{N} \backslash B(0,1)} \tilde{I}_{\alpha, k}(x, y) f(y) d y
$$

for a locally integrable function $f$ on $\mathbf{R}^{N}$.

The following estimates are fundamental (see [32], [33] and [49]).

LEMmA 13. (1) If $2|x|<|y|$, then $\left|\tilde{I}_{\alpha, k}(x, y)\right| \leq C|x|^{k}|y|^{\alpha-N-k}$.

(2) If $|x| / 2 \leq|y| \leq 2|x|$, then $\left|\tilde{I}_{\alpha, k}(x, y)\right| \leq C|x-y|^{\alpha-N}$.

(3) If $1 \leq|y| \leq|x| / 2$, then $\left|\tilde{I}_{\alpha, k}(x, y)\right| \leq C|x|^{k-1}|y|^{\alpha-N-(k-1)}$. 
Lemma 14. Assume that $\Phi_{\infty}(t)$ satisfies $\left(\Phi_{\infty} 2\right)$ and $\left(\Phi_{\infty} \omega 1 ; k-\alpha\right)$ for $\varepsilon_{1}>0$. Then, for $0<\varepsilon<\varepsilon_{1}$, there exists a constant $C>0$ such that, for all $x \in B(0,2 r)$ with $r \geq 1$ and nonnegative functions $f \in L_{l o c}^{1}\left(\mathbf{R}^{N}\right)$,

$$
\begin{aligned}
& \left|\tilde{I}_{\alpha, k}\left(f \chi_{\mathbf{R}^{N} \backslash B(0,4 r)}\right)(x)\right| \\
& \quad \leq C r^{\varepsilon+\alpha} \omega(r)^{-1} \Phi_{\infty}^{-1}\left(r^{-N}\right)\left(\int_{r}^{\infty}\left(t^{-\varepsilon} \omega(t)\|f\|_{L^{\Phi}(A(0, t))}\right)^{q} \frac{d t}{t}\right)^{1 / q} .
\end{aligned}
$$

Proof. Let $f \in L_{l o c}^{1}\left(\mathbf{R}^{N}\right)$ be nonnegative, $r \geq 1$ and $x \in B(0,2 r)$. By Lemma 13 (1),

$$
\begin{aligned}
\left|\tilde{I}_{\alpha, k}\left(f \chi_{\mathbf{R}^{N} \backslash B(0,4 r)}\right)(x)\right| & \leq C|x|^{k} \int_{\mathbf{R}^{N} \backslash B(0,4 r)}|y|^{\alpha-N-k} f(y) d y \\
& \leq C r^{\alpha} H_{k-\alpha}^{\infty} f(2 r),
\end{aligned}
$$

so that we obtain the required inequality by Lemma 7 .

Lemma 15. Assume $\Phi_{\infty}(t)$ satisfies $\left(\Phi_{\infty} 2\right)$ and $\left(\Phi_{\infty} \omega 2 ; k-1-\alpha\right)$ for $\varepsilon_{2}>0$. Then, for $0<\varepsilon<\varepsilon_{2}$, there exists a constant $C>0$ such that for all $x \in B(0,2 r)$ with $r \geq 1$ and nonnegative functions $f \in L_{\text {loc }}^{1}\left(\mathbf{R}^{N}\right)$,

$$
\begin{aligned}
& \left|\tilde{I}_{\alpha, k}\left(f \chi_{B(0,|x| / 2)}\right)(x)\right| \\
& \quad \leq C r^{-\varepsilon+\alpha} \omega(r)^{-1} \Phi_{\infty}^{-1}\left(r^{-N}\right)\left(\int_{1 / 2}^{r}\left(t^{\varepsilon} \omega(t)\|f\|_{L^{\Phi}(A(0, t))}\right)^{q} \frac{d t}{t}\right)^{1 / q} .
\end{aligned}
$$

Proof. Let $f \in L_{l o c}^{1}\left(\mathbf{R}^{N}\right)$ be nonnegative, $r \geq 1$ and $x \in B(0,2 r)$. By Lemma 13 (3),

$$
\begin{aligned}
\left|\tilde{I}_{\alpha, k}\left(f \chi_{B(0,|x| / 2)}\right)(x)\right| & \leq C|x|^{k-1} \int_{B(0,|x| / 2) \backslash B(0,1)}|y|^{\alpha-N-(k-1)} f(y) d y \\
& \leq C r^{\alpha} H_{k-1-\alpha}^{0} f(r)
\end{aligned}
$$

so that we obtain the required inequality by Lemma 8 .

Now we give the Sobolev type inequality for generalized Riesz potentials of functions in $\mathscr{H}^{\Phi, q, \omega}\left(\mathbf{R}^{N}\right)$.

THEOREM 5. Suppose $\Phi(x, t)$ satisfies $(\Phi 3 ; 0 ; p),(\Phi 3 ; \infty ; q),(\Phi 5 ; \eta),(\Phi 6 ; \tau)$ and $(\Phi \alpha)$ for $p>1, q>1, \eta>0$ and $\tau>0$ satisfying $\eta \leq q / N$ and $\tau \leq p$. Assume that $\Psi(x, t)$ satisfies $(\Psi \Phi \alpha)$. Assume $\Phi_{\infty}(t)$ satisfies $\left(\Phi_{\infty} 2^{\prime}\right), \quad\left(\Phi_{\infty} \omega 1\right.$; 
$k-\alpha)$ and $\left(\Phi_{\infty} \omega 2 ; k-1-\alpha\right)$. Then there exists a constant $C>0$ such that

$$
\left\|I_{\alpha, k} f\right\|_{\mathscr{H}^{\Psi, q, \omega}\left(\mathbf{R}^{N}\right)} \leq C\|f\|_{\mathscr{H}^{\Phi, q, \omega}\left(\mathbf{R}^{N}\right)}
$$

for all $f \in \mathscr{H}^{\Phi, q, \omega}\left(\mathbf{R}^{N}\right)$.

Proof. Let $f$ be a nonnegative measurable function on $\mathbf{R}^{N}$ such that $\|f\|_{\mathscr{H}^{\Phi, q, \omega}\left(\mathbf{R}^{N}\right)} \leq 1$. For $r \geq 2$ and fixed $x \in A(0, r)$, set

$$
\begin{aligned}
f & =f \chi_{B(0,1)}+f \chi_{B(0,|x| / 2) \backslash B(0,1)}+f \chi_{B(0,4 r) \backslash B(0,|x| / 2)}+f \chi_{\mathbf{R}^{N} \backslash B(0,4 r)} \\
& =f_{0}+f_{1, x}+f_{2, r, x}+f_{3, r} .
\end{aligned}
$$

For $f_{0}$, we note

$$
I_{\alpha, k} f_{0}(x)=I_{\alpha} f_{0}(x) \leq C|x|^{\alpha-N} .
$$

For $f_{2, r, x}$, by Lemma $13(1)$, (2), we see that

$$
\left|I_{\alpha, k} f_{2, r, x}(x)\right|=\left|\tilde{I}_{\alpha, k} f_{2, r, x}(x)\right| \leq C I_{\alpha}\left(f \chi_{B(0,4 r) \backslash B(0, r / 2)}\right)(x) .
$$

Since $I_{\alpha, k} f_{1, x}=\tilde{I}_{\alpha, k}\left(f \chi_{B(0,|x| / 2)}\right)$ and $I_{\alpha, k} f_{3, r}=\tilde{I}_{\alpha, k}\left(f \chi_{\mathbf{R}^{N} \backslash B(0,4 r)}\right)$, it follows from Lemmas 15 and 14 that

$$
\begin{aligned}
I_{\alpha, k} f(x) \leq C & \left\{|x|^{\alpha-N}+I_{\alpha}\left(f \chi_{B(0,4 r) \backslash B(0, r / 2)}\right)(x)\right. \\
& +r^{-\varepsilon_{2}^{\prime}+\alpha} \omega(r)^{-1} \Phi_{\infty}^{-1}\left(r^{-N}\right)\left(\int_{1 / 2}^{r}\left(t^{\varepsilon^{\prime}} \omega(t)\|f\|_{L^{\Phi}(A(0, t))}\right)^{q} \frac{d t}{t}\right)^{1 / q} \\
& \left.+r^{\varepsilon_{1}^{\prime}+\alpha} \omega(r)^{-1} \Phi_{\infty}^{-1}\left(r^{-N}\right)\left(\int_{r}^{\infty}\left(t^{-\varepsilon_{1}^{\prime}} \omega(t)\|f\|_{L^{\Phi}(A(0, t))}\right)^{q} \frac{d t}{t}\right)^{1 / q}\right\}
\end{aligned}
$$

for $x \in A(0, r)$, with $0<\varepsilon_{1}^{\prime}<\varepsilon_{1}$ and $0<\varepsilon_{2}^{\prime}<\varepsilon_{2}$.

Then, we obtain

$$
\int_{2}^{\infty}\left(\omega(r)\left\|I_{\alpha, k} f\right\|_{L^{\Psi}(A(0, r))}\right)^{q} \frac{d r}{r} \leq C
$$

by the same arguments as in the proof of Theorem 4 .

By Lemma 13, we see that $\left|\tilde{I}_{\alpha, k}(x, y)\right| \leq C I_{\alpha}(x-y)$ for $|x| \leq 4$ and $|y| \geq 1$. Hence, as in the proof of Theorem 4 we can show that $\left\|I_{\alpha, k} f\right\|_{L^{\Psi}(B(0,4))} \leq C$, which implies

$$
\left\|I_{\alpha, k} f\right\|_{L^{\Psi}(B(0,2))}+\int_{1}^{2}\left(\omega(r)\left\|I_{\alpha, k} f\right\|_{L^{\Psi}(A(0, r))}\right)^{q} \frac{d r}{r} \leq C .
$$


REMARK 15. Let $\Phi$ be as in Example 1 and let $\Phi_{\infty}(t)$ be as in Remark 8 . If $\omega(r)=r^{v}$, then $\left(\Phi_{\infty} \omega 1 ; k-\alpha\right)$ and $\left(\Phi_{\infty} \omega 2 ; k-1-\alpha\right)$ hold when

$$
\alpha-N / p_{1}(\infty)-v<k<\alpha-N / p_{1}(\infty)-v+1 \text {. }
$$

7. $\underline{\mathscr{H}}^{\Phi, q, \omega}\left(\mathbf{R}^{N}\right)$ and $\overline{\mathscr{H}}^{\Phi, q, \omega}\left(\mathbf{R}^{N}\right)$

We further consider the space $\underline{\mathscr{H}}^{\Phi, q, \omega}\left(\mathbf{R}^{N}\right)$ of locally integrable functions $f$ on $\mathbf{R}^{N}$ satisfying

$$
\|f\|_{\mathscr{H}^{\Phi, q, \omega}\left(\mathbf{R}^{N}\right)}=\left(\int_{1}^{\infty}\left(\omega(r)\|f\|_{L^{\Phi}(B(0, r))}\right)^{q} \frac{d r}{r}\right)^{1 / q}<\infty
$$

and the space $\overline{\mathscr{H}}^{\Phi, q, \omega}\left(\mathbf{R}^{N}\right)$ consisting of all measurable functions $f$ on $\mathbf{R}^{N}$ satisfying

$$
\|f\|_{\overline{\mathscr{H}}^{\Phi, q, \omega}\left(\mathbf{R}^{N}\right)}=\|f\|_{L^{\Phi}(B(0,2))}+\left(\int_{1}^{\infty}\left(\omega(r)\|f\|_{L^{\Phi}\left(\mathbf{R}^{N} \backslash B(0, r)\right)}\right)^{q} \frac{d r}{r}\right)^{1 / q}<\infty .
$$

If $\omega(r)$ satisfies

$$
\text { ( } \omega 3) \quad \int_{1}^{\infty} \omega(r)^{q} \frac{d r}{r}<\infty
$$

then

$$
L^{\Phi}\left(\mathbf{R}^{N}\right)=\overline{\mathscr{H}}^{\Phi, q, \omega}\left(\mathbf{R}^{N}\right) \hookrightarrow \underline{\mathscr{H}}^{\Phi, q, \omega}\left(\mathbf{R}^{N}\right) \hookrightarrow \mathscr{H}^{\Phi, q, \omega}\left(\mathbf{R}^{N}\right)
$$

and if $\omega$ satisfies

then

$$
(\omega 4) \quad \int_{1}^{\infty} \omega(r)^{q} \frac{d r}{r}=\infty
$$

$$
\{0\}=\underline{\mathscr{H}}^{\Phi, q, \omega}\left(\mathbf{R}^{N}\right) \subset \overline{\mathscr{H}}^{\Phi, q, \omega}\left(\mathbf{R}^{N}\right) \hookrightarrow \mathscr{H}^{\Phi, q, \omega}\left(\mathbf{R}^{N}\right) \cap L^{\Phi}\left(\mathbf{R}^{N}\right) .
$$

Therefore, it is natural to assume $(\omega 3)$ when we treat the space $\underline{\mathscr{H}}^{\Phi, q, \omega}\left(\mathbf{R}^{N}\right)$; and we assume $(\omega 4)$ when we treat the space $\overline{\mathscr{H}}^{\Phi, q, \omega}\left(\mathbf{R}^{N}\right)$.

Proposition 1. (1) Suppose $\omega(r)$ satisfies

( $\omega 5 a) \quad r \mapsto r^{a} \omega(r)$ is almost decreasing on $[1, \infty)$ for some $a>0$. Then, $\underline{\mathscr{H}}^{\Phi, q, \omega}\left(\mathbf{R}^{N}\right)=\mathscr{H}^{\Phi, q, \omega}\left(\mathbf{R}^{N}\right)$.

(2) Suppose $\omega(r)$ satisfies

$(\omega 5 b) \quad r \mapsto r^{-b} \omega(r)$ is almost increasing on $[1, \infty)$ for some $b>0$. Then, $\overline{\mathscr{H}}^{\Phi, q, \omega}\left(\mathbf{R}^{N}\right)=\mathscr{H}^{\Phi, q, \omega}\left(\mathbf{R}^{N}\right)$.

Proof. (1) Assume $(\omega 5 a)$. Let $X=\mathscr{H}^{\Phi, q, \omega}\left(\mathbf{R}^{N}\right)$ and $Y=\underline{\mathscr{H}}^{\Phi, q, \omega}\left(\mathbf{R}^{N}\right)$. Since $Y \hookrightarrow X$ in general, we have to show $X \hookrightarrow Y$. 
Let $f \in X$ and let $K$ be a compact set in $\mathbf{R}^{N}$. Then note from Lemma 2 and $(\omega 5 a)((\omega 5 a)$ implies $(\omega 3))$ that $\left\|f \chi_{K}\right\|_{Y}<\infty$. Set $F(r)=\left\|f \chi_{K}\right\|_{L^{\Phi}(A(0, r))}$ and $G(r)=\left\|f \chi_{K}\right\|_{L^{\Phi}(B(0, r))}$. Then

$$
G(2 r) \leq F(r)+G(r) .
$$

Set $\omega_{*}(r)=r^{-a} \inf _{1 \leq s \leq r} s^{a} \omega(s)$ for $r \geq 1$. Then

$$
\omega_{*}(r) \leq \omega(r) \leq C_{0} \omega_{*}(r)
$$

by $(\omega 5 a)$ with a constant $C_{0} \geq 1$.

Set

$$
\begin{gathered}
A=\left(\int_{1}^{\infty}\left(\omega_{*}(r) F(r)\right)^{q} \frac{d r}{r}\right)^{1 / q} \\
B_{1}=\left(\int_{1}^{2}\left(\omega_{*}(r) G(r)\right)^{q} \frac{d r}{r}\right)^{1 / q} \text { and } B_{2}=\left(\int_{2}^{\infty}\left(\omega_{*}(r) G(r)\right)^{q} \frac{d r}{r}\right)^{1 / q} .
\end{gathered}
$$

All of these are finite values.

By (20)

$$
\left(\int_{1}^{\infty}\left(\omega_{*}(r) G(2 r)\right)^{q} \frac{d r}{r}\right)^{1 / q} \leq \begin{cases}A+\left(B_{1}^{q}+B_{2}^{q}\right)^{1 / q} & \text { if } q \geq 1 \\ \left(A^{q}+B_{1}^{q}+B_{2}^{q}\right)^{1 / q} & \text { if } 0<q<1\end{cases}
$$

Since $r^{a} \omega_{*}(r)$ is decreasing, $\omega_{*}(r / 2) \geq 2^{a} \omega_{*}(r)$ for $r \geq 2$, so that

$$
\int_{1}^{\infty}\left(\omega_{*}(r) G(2 r)\right)^{q} \frac{d r}{r}=\int_{2}^{\infty}\left(\omega_{*}(r / 2) G(r)\right)^{q} \frac{d r}{r} \geq 2^{a q} B_{2}^{q} .
$$

Hence

$$
2^{a} B_{2} \leq \begin{cases}A+\left(B_{1}^{q}+B_{2}^{q}\right)^{1 / q} & \text { if } q \geq 1 \\ \left(A^{q}+B_{1}^{q}+B_{2}^{q}\right)^{1 / q} & \text { if } 0<q<1\end{cases}
$$

which implies

$$
\left(B_{1}^{q}+B_{2}^{q}\right)^{1 / q} \leq C\left(A+2^{a} B_{1}\right)
$$

with $C>0$ depending only on $a$ and $q$. Note that $B_{1} \leq C_{1}\left\|f \chi_{K}\right\|_{L^{\Phi}(B(0,2))}$ with $C_{1}=\omega(1)(\log 2)^{1 / q}$. By (21) $\left\|f \chi_{K}\right\|_{Y} \leq C_{0}\left(B_{1}^{q}+B_{2}^{q}\right)^{1 / q}$ and $\left\|f \chi_{K}\right\|_{X} \geq$ $A+\left\|f \chi_{K}\right\|_{L^{\Phi}(B(0,2))}$. Hence (22) implies

$$
\left\|f \chi_{K}\right\|_{Y} \leq C\left\|f \chi_{K}\right\|_{X}
$$

with a constant $C>0$ independent of $K$. By the monotone convergence theorem, we obtain the required result. 
(2) Assume $(\omega 5 b)$. Let $X$ be as above and $Z=\overline{\mathscr{H}}^{\Phi, q, \omega}\left(\mathbf{R}^{N}\right)$. Since $Z \hookrightarrow X$ in general, we shall show $X \hookrightarrow Z$.

Let $f \in X$ and let $K$ be a compact set in $\mathbf{R}^{N}$. Then note from Lemma 2 that $\left\|f \chi_{K}\right\|_{Z}<\infty$. Set $F(r)=\left\|f \chi_{K}\right\|_{L^{\Phi}(A(0, r))}$ and $H(r)=\left\|f \chi_{K}\right\|_{L^{\Phi}\left(\mathbf{R}^{N} \backslash B(0, r)\right)}$. Then

$$
H(r) \leq F(r)+H(2 r) .
$$

Set $\omega^{*}(r)=r^{b} \sup _{1 \leq s \leq r} s^{-b} \omega(s)$ for $r \geq 1$. Then

$$
\omega(r) \leq \omega^{*}(r) \leq C_{2} \omega(r)
$$

by $(\omega 5 b)$ with a constant $C_{2} \geq 1$. Since $r^{-b} \omega^{*}(r)$ is increasing, $\omega^{*}(r / 2) \leq$ $2^{-b} \omega^{*}(r)$ for $r \geq 2$, so that

$$
\int_{1}^{\infty}\left(\omega^{*}(r) H(2 r)\right)^{q} \frac{d r}{r} \leq 2^{-b q} \int_{2}^{\infty}\left(\omega^{*}(r) H(r)\right)^{q} \frac{d r}{r}
$$

Hence, by (23), we have

$$
\int_{1}^{\infty}\left(\omega^{*}(r) H(r)\right)^{q} \frac{d r}{r} \leq C \int_{1}^{\infty}\left(\omega^{*}(r) F(r)\right)^{q} \frac{d r}{r}
$$

which implies $\left\|f \chi_{K}\right\|_{Z} \leq C\left\|f \chi_{K}\right\|_{X}$ in view of (24) with a constant $C>0$ independent of $K$. Hence, by the monotone convergence theorem, we obtain the required result.

The following example shows that there are $\omega(r)$ satisfying $(\omega 3)$ for which $L^{\Phi}\left(\mathbf{R}^{N}\right) \neq \underline{\mathscr{H}}^{\Phi, q, \omega}\left(\mathbf{R}^{N}\right) \neq \mathscr{H}^{\Phi, q, \omega}\left(\mathbf{R}^{N}\right)$; and also there are $\omega(r)$ satisfying $(\omega 4)$ for which $\{0\} \neq \overline{\mathscr{H}}^{\Phi, q, \omega}\left(\mathbf{R}^{N}\right) \neq \mathscr{H}^{\Phi, q, \omega}\left(\mathbf{R}^{N}\right)$.

ExAmPle 3. Let $\Phi(x, t)=t^{p}, p \geq 1$ and

$$
\omega(r) \sim \log (e+r)^{v}, \quad v \in \mathbf{R} .
$$

(1) If $v<-1 / q$, then $\omega(r)$ satisfies $(\omega 3)$ and

$$
L^{p}\left(\mathbf{R}^{N}\right) \neq \underline{\mathscr{H}}^{\Phi, q, \omega}\left(\mathbf{R}^{N}\right) \neq \mathscr{H}^{\Phi, q, \omega}\left(\mathbf{R}^{N}\right) .
$$

(2) If $v \geq-1 / q$, then $\omega(r)$ satisfies $(\omega 4)$ and

$$
\{0\} \neq \overline{\mathscr{H}}^{\Phi, q, \omega}\left(\mathbf{R}^{N}\right) \neq \mathscr{H}^{\Phi, q, \omega}\left(\mathbf{R}^{N}\right) .
$$

In fact, consider the function

$$
f_{a}(x)=|x|^{-N / p}(\log (e+|x|))^{-a} \chi_{\mathbf{R}^{N} \backslash B(0,2)}(x)
$$

for $a \in \mathbf{R}$. Then, $\left\|f_{a}\right\|_{L^{p}(A(0, r))} \sim(\log (e+r))^{-a}$ for $r \geq 2$, so that

$$
f_{a} \in \mathscr{H}^{\Phi, q, \omega}\left(\mathbf{R}^{N}\right) \quad \text { if (and only if) } a>v+1 / q .
$$


On the other hand, for $r \geq 3,\left\|f_{a}\right\|_{L^{p}(B(0, r))} \sim(\log (e+r))^{-a+1 / p}$ in case $a<1 / p$, $\sim(\log (\log (e+r)))^{1 / p}$ in case $a=1 / p$ and $\sim C$ in case $a>1 / p$, so that

$$
f_{a} \in \underline{\mathscr{H}}^{\Phi, q, \omega}\left(\mathbf{R}^{N}\right) \quad \text { if and only if } a>v+1 / p+1 / q
$$

when $v<-1 / q$. Thus, $f_{a} \in \mathscr{H}^{\Phi, q, \omega}\left(\mathbf{R}^{N}\right) \backslash \underline{\mathscr{H}}^{\Phi, q, \omega}\left(\mathbf{R}^{N}\right)$ if $v+1 / q<a \leq v+$ $1 / p+1 / q$ when $v<-1 / q$.

Since $f_{a} \in L^{p}\left(\mathbf{R}^{N}\right)$ if and only if $a p>1, f_{a} \in \underline{\mathscr{H}}^{\Phi, q, \omega}\left(\mathbf{R}^{N}\right) \backslash L^{p}\left(\mathbf{R}^{N}\right)$ if $v+1 / p+1 / q<a \leq 1 / p$. Such $a$ exists when $v<-1 / q$.

Next, for $r \geq 2,\left\|f_{a}\right\|_{L^{p}\left(\mathbf{R}^{N} \backslash B(0, r)\right)} \sim(\log (e+r))^{-a+1 / p}$ in case $a>1 / p$ and $=\infty$ in case $a \leq 1 / p$. Hence, in case $v \geq-1 / q, f_{a} \in \mathscr{H}^{\Phi, q, \omega}\left(\mathbf{R}^{N}\right) \backslash \overline{\mathscr{H}} \Phi, q, \omega\left(\mathbf{R}^{N}\right)$ if $v+1 / q<a \leq v+1 / p+1 / q$. Since $\chi_{B(0,1)} \in \overline{\mathscr{H}}^{\Phi, q, \omega}\left(\mathbf{R}^{N}\right), \quad \overline{\mathscr{H}}^{\Phi, q, \omega}\left(\mathbf{R}^{N}\right) \neq$ $\{0\}$.

REMARK 16. Since $\underline{\mathscr{H}}^{\Phi, q, \omega}\left(\mathbf{R}^{N}\right) \hookrightarrow \mathscr{H}^{\Phi, q, \omega}\left(\mathbf{R}^{N}\right)$, the second inequality (3) also holds with $\|f\|_{\mathscr{H}^{\Phi, q, \omega}\left(\mathbf{R}^{N}\right)}$ replaced by $\|f\|_{\mathscr{H}^{\Phi, q, \omega}\left(\mathbf{R}^{N}\right)}$.

Analogous inequality is trivial for $\|f\|_{\overline{\mathscr{H}}^{\Phi, q, \omega}\left(\mathbf{R}^{N}\right)}$, since $\overline{\mathscr{H}}^{\Phi, q, \omega}\left(\mathbf{R}^{N}\right) \hookrightarrow$ $L^{\Phi}\left(\mathbf{R}^{N}\right)$.

For the boundedness of the maximal operator, we have the following results (cf. [9]).

THEOREM 6. Suppose that $\Phi(x, t)$ satisfies $(\Phi 3 ; 0 ; p),(\Phi 3 ; \infty ; q),(\Phi 5 ; \eta)$ and $(\Phi 6 ; \tau)$ for $p>1, q>1, \eta>0$ and $\tau>0$ satisfying $\eta \leq q / N$ and $\tau \leq p$. Assume that $\Phi_{\infty}(t)$ satisfies $\left(\Phi_{\infty} 2\right)$ and $\left(\Phi_{\infty} \omega 1 ; 0\right)$. Then the maximal operator $M$ is bounded from $\underline{\mathscr{H}}^{\Phi, q, \omega}\left(\mathbf{R}^{N}\right)$ to itself.

Proof. Let $f$ be a nonnegative measurable function on $\mathbf{R}^{N}$ such that $\|f\|_{\mathscr{H}^{\Phi, q, \omega}\left(\mathbf{R}^{N}\right)} \leq 1$. For $r \geq 1$, set

$$
f=f \chi_{B(0,2 r)}+f \chi_{\mathbf{R}^{N} \backslash B(0,2 r)}=g_{1, r}+g_{2, r}
$$

By Lemma 6,

$$
\left\|M g_{1, r}\right\|_{L^{\Phi}(B(0, r))} \leq C\left\|g_{1, r}\right\|_{L^{\Phi}\left(\mathbf{R}^{N}\right)}=C\|f\|_{L^{\Phi}(B(0,2 r))},
$$

so that

$$
\int_{1}^{\infty}\left(\omega(r)\left\|M g_{1, r}\right\|_{L^{\Phi}(B(0, r))}\right)^{q} \frac{d r}{r} \leq C \int_{1}^{\infty}\left(\omega(r)\|f\|_{L^{\Phi}(B(0,2 r))}\right)^{q} \frac{d r}{r} \leq C .
$$

For $g_{2, r}$, we argue as for $f_{3, r}$ in the proof of Theorem 1 to obtain

$$
M g_{2, r}(x) \leq C r^{\varepsilon_{1}^{\prime}} \omega(r)^{-1} \Phi_{\infty}^{-1}\left(r^{-N}\right)\left(\int_{r}^{\infty}\left(t^{-\varepsilon_{1}^{\prime}} \omega(t)\|f\|_{L^{\Phi}(A(0, t))}\right)^{q} \frac{d t}{t}\right)^{1 / q}
$$


for $x \in B(0, r)$ with $0<\varepsilon_{1}^{\prime}<\varepsilon_{1}$, which implies

$$
\int_{1}^{\infty}\left(\omega(r)\left\|M g_{2, r}\right\|_{L^{\Phi}(B(0, r))}\right)^{q} \frac{d r}{r} \leq C \int_{1}^{\infty}\left(\omega(r)\|f\|_{L^{\Phi}(B(0,2 r))}\right)^{q} \frac{d r}{r} \leq C .
$$

THEOREM 7. Suppose that $\Phi(x, t)$ satisfies $(\Phi 3 ; 0 ; p),(\Phi 3 ; \infty ; q),(\Phi 5 ; \eta)$ and $(\Phi 6 ; \tau)$ for $p>1, q>1, \eta>0$ and $\tau>0$ satisfying $\eta \leq q / N$ and $\tau \leq p$. Assume that $\Phi_{\infty}(t)$ satisfies $\left(\Phi_{\infty} 2\right)$ and $\left(\Phi_{\infty} \omega 2 ;-N\right)$. Then the maximal operator $M$ is bounded from $\overline{\mathscr{H}}^{\Phi, q, \omega}\left(\mathbf{R}^{N}\right)$ to itself.

Proof. Let $f$ be a nonnegative measurable function on $\mathbf{R}^{N}$ such that $\|f\|_{\overline{\mathscr{H}}^{\Phi, q, \omega}\left(\mathbf{R}^{N}\right)} \leq 1$. Then $\|f\|_{L^{\Phi}\left(\mathbf{R}^{N}\right)} \leq C$.

For $r \geq 2$, set

$$
f=f \chi_{B(0,1)}+f \chi_{B(0, r / 2) \backslash B(0,1)}+f \chi_{\mathbf{R}^{N} \backslash B(0, r / 2)}=f_{0}+f_{1, r}+h_{2, r} .
$$

By (5), we see that

$$
\left\|M f_{0}\right\|_{L^{\Phi}\left(\mathbf{R}^{N} \backslash B(0, r)\right)} \leq C r^{-N}\left\{\Phi_{\infty}^{-1}\left(r^{-N}\right)\right\}^{-1}
$$

and using $\left(\Phi_{\infty} \omega 2 ;-N\right)$, we have

$$
\int_{2}^{\infty}\left(\omega(r)\left\|M f_{0}\right\|_{L^{\Phi}\left(\mathbf{R}^{N} \backslash B(0, r)\right)}\right)^{q} \frac{d r}{r} \leq C
$$

by the same arguments as to obtain (6) in the proof of Theorem 1 .

In view of (7) in the proof of Theorem 1, we see

$$
\begin{aligned}
& \int_{2}^{\infty}\left(\omega(r)\left\|M f_{1, r}\right\|_{L^{\Phi}\left(\mathbf{R}^{N} \backslash B(0, r)\right)}\right)^{q} \frac{d r}{r} \\
& \quad \leq C \int_{1 / 2}^{\infty}\left(\omega(r)\|f\|_{L^{\Phi}(A(0, r))}\right)^{q} \frac{d r}{r} \\
& \quad \leq C\left\{\|f\|_{L^{\Phi}(B(0,2))}+\int_{1}^{\infty}\left(\omega(r)\|f\|_{L^{\Phi}\left(\mathbf{R}^{N} \backslash B(0, r)\right)}\right)^{q} \frac{d r}{r}\right\} \\
& \quad \leq C .
\end{aligned}
$$

By Lemma 6,

$$
\left\|M h_{2, r}\right\|_{L^{\Phi}\left(\mathbf{R}^{N} \backslash B(0, r)\right)} \leq C\left\|h_{2, r}\right\|_{L^{\Phi}\left(\mathbf{R}^{N}\right)}=C\|f\|_{L^{\Phi}\left(\mathbf{R}^{N} \backslash B(0, r)\right)},
$$

so that

$$
\begin{aligned}
\int_{2}^{\infty}\left(\omega(r)\left\|M h_{2, r}\right\|_{L^{\Phi}\left(\mathbf{R}^{N} \backslash B(0, r)\right)}\right)^{q} \frac{d r}{r} & \leq C \int_{2}^{\infty}\left(\omega(r)\|f\|_{L^{\Phi}\left(\mathbf{R}^{N} \backslash B(0, r)\right)}\right)^{q} \frac{d r}{r} \\
& \leq C .
\end{aligned}
$$


Thus,

$$
\int_{2}^{\infty}\left(\omega(r)\|M f\|_{L^{\Phi}\left(\mathbf{R}^{N} \backslash B(0, r)\right)}\right)^{q} \frac{d r}{r} \leq C
$$

Finally, since $\|M f\|_{L^{\Phi}\left(\mathbf{R}^{N}\right)} \leq C\|f\|_{L^{\Phi}\left(\mathbf{R}^{N}\right)} \leq C$ by Lemma 6 ,

$$
\|M f\|_{L^{\Phi}(B(0,2))}+\int_{1}^{2}\left(\omega(r)\|M f\|_{L^{\Phi}\left(\mathbf{R}^{N} \backslash B(0, r)\right)}\right)^{q} \frac{d r}{r} \leq C .
$$

As to Sobolev's inequalities, we have the following results (see also [10]).

TheOrem 8. Suppose $\Phi(x, t)$ satisfies $(\Phi 3 ; 0 ; p),(\Phi 3 ; \infty ; q),(\Phi 5 ; \eta),(\Phi 6 ; \tau)$ and $(\Phi \alpha)$ for $p>1, q>1, \eta>0$ and $\tau>0$ satisfying $\eta \leq q / N$ and $\tau \leq p$. Assume that $\Psi(x, t)$ satisfies $(\Psi \Phi \alpha)$. For the function $\Phi_{\infty}(t)$, assume $\left(\Phi_{\infty} 2^{\prime}\right)$ and $\left(\Phi_{\infty} \omega 1 ;-\alpha\right)$ hold. Then there exists a constant $C>0$ such that

$$
\left\|I_{\alpha} f\right\|_{\mathscr{\mathscr { P }}^{\Psi, q, \omega}\left(\mathbf{R}^{N}\right)} \leq C\|f\|_{\mathscr{\mathscr { H }}^{\Phi, q, \omega}\left(\mathbf{R}^{N}\right)}
$$

for all $f \in \underline{\mathscr{H}}^{\Phi, q, \omega}\left(\mathbf{R}^{N}\right)$.

Proof. Let $f$ be a nonnegative measurable function on $\mathbf{R}^{N}$ such that $\|f\|_{\mathscr{H}^{\Phi, q, \omega}\left(\mathbf{R}^{N}\right)} \leq 1$. For $r \geq 1$, set

$$
f=f \chi_{B(0,2 r)}+f \chi_{\mathbf{R}^{N} \backslash B(0,2 r)}=g_{1, r}+g_{2, r}
$$

By Lemma 12,

$$
\left\|I_{\alpha} g_{1, r}\right\|_{L^{\Psi}(B(0, r))} \leq C\left\|g_{1, r}\right\|_{L^{\Phi}\left(\mathbf{R}^{N}\right)}=C\|f\|_{L^{\Phi}(B(0,2 r))},
$$

so that

$$
\int_{1}^{\infty}\left(\omega(r)\left\|I_{\alpha} g_{1, r}\right\|_{L^{\Phi}(B(0, r))}\right)^{q} \frac{d r}{r} \leq C \int_{1}^{\infty}\left(\omega(r)\|f\|_{L^{\Phi}(B(0,2 r))}\right)^{q} \frac{d r}{r} \leq C .
$$

For $g_{2, r}$, we argue as for $f_{3, r}$ in the proof of Theorem 4 to obtain

$$
\left\|I_{\alpha} g_{2, r}\right\|_{L^{\Psi}(B(0, r))} \leq C r^{\varepsilon_{1}^{\prime}} \omega(r)^{-1}\left(\int_{r}^{\infty}\left(t^{-\varepsilon_{1}^{\prime}} \omega(t)\|f\|_{L^{\Phi}(A(0, t))}\right)^{q} \frac{d t}{t}\right)^{1 / q}
$$

for $0<\varepsilon_{1}^{\prime}<\varepsilon_{1}$, which implies

$$
\int_{1}^{\infty}\left(\omega(r)\left\|I_{\alpha} g_{2, r}\right\|_{L^{\Psi}(B(0, r))}\right)^{q} \frac{d r}{r} \leq C \int_{1}^{\infty}\left(\omega(r)\|f\|_{L^{\Phi}(B(0,2 r))}\right)^{q} \frac{d r}{r} \leq C .
$$

THEOREM 9. Suppose $\Phi(x, t)$ satisfies $(\Phi 3 ; 0 ; p),(\Phi 3 ; \infty ; q),(\Phi 5 ; \eta),(\Phi 6 ; \tau)$ and $(\Phi \alpha)$ for $p>1, q>1, \eta>0$ and $\tau>0$ satisfying $\eta \leq q / N$ and $\tau \leq p$. Assume that $\Psi(x, t)$ satisfies $(\Psi \Phi \alpha)$. For the function $\Phi_{\infty}(t)$, assume $\left(\Phi_{\infty} 2^{\prime}\right)$ and 
$\left(\Phi_{\infty} \omega 2 ;-N\right)$ hold. Then there exists a constant $C>0$ such that

$$
\left\|I_{\alpha} f\right\|_{\overline{\mathscr{H}}^{\Psi, q, \omega}\left(\mathbf{R}^{N}\right)} \leq C\|f\|_{\overline{\mathscr{H}}^{\Phi, q, \omega}\left(\mathbf{R}^{N}\right)}
$$

for all $f \in \overline{\mathscr{H}}^{\Phi, q, \omega}\left(\mathbf{R}^{N}\right)$.

Proof. Let $f$ be a nonnegative measurable function on $\mathbf{R}^{N}$ such that $\|f\|_{\overline{\mathscr{H}}^{\Phi, q, \omega}\left(\mathbf{R}^{N}\right)} \leq 1$ and for $r \geq 2$, set

$$
f=f \chi_{B(0,1)}+f \chi_{B(0, r / 2) \backslash B(0,1)}+f \chi_{\mathbf{R}^{N} \backslash B(0, r / 2)}=f_{0}+f_{1, r}+h_{2, r} .
$$

Since $\int_{B(0,1)} f(y) d y \leq C\|f\|_{L^{\Phi}(B(0,1))} \leq C$, we see that

$$
\left\|I_{\alpha} f_{0}\right\|_{L^{\Psi}\left(\mathbf{R}^{N} \backslash B(0, r)\right)} \leq C r^{-N}\left\{\Phi_{\infty}^{-1}\left(r^{-N}\right)\right\}^{-1},
$$

and hence

$$
\int_{2}^{\infty}\left(\omega(r)\left\|I_{\alpha} f_{0}\right\|_{L^{{ }}\left(\mathbf{R}^{N} \backslash B(0, r)\right)}\right)^{q} \frac{d r}{r} \leq C
$$

in the same way as in the proof of Theorem 4 .

Also, as in the proof of Theorem 4, we see

$$
\int_{2}^{\infty}\left(\omega(r)\left\|I_{\alpha} f_{1, r}\right\|_{L^{\Psi}\left(\mathbf{R}^{N} \backslash B(0, r)\right)}\right)^{q} \frac{d r}{r} \leq C .
$$

For $h_{2, r}$, we use Lemma 12 to obtain

$$
\left\|I_{\alpha} h_{2, r}\right\|_{L^{\Psi}\left(\mathbf{R}^{N} \backslash B(0, r)\right)} \leq C\left\|h_{2, r}\right\|_{L^{\Phi}\left(\mathbf{R}^{N}\right)}=C\|f\|_{L^{\Phi}\left(\mathbf{R}^{N} \backslash B(0, r / 2)\right)},
$$

which implies

$$
\int_{2}^{\infty}\left(\omega(r)\left\|I_{\alpha} h_{2, r}\right\|_{L^{\Psi}\left(\mathbf{R}^{N} \backslash B(0, r)\right)}\right)^{q} \frac{d r}{r} \leq C
$$

Finally, since $\left\|I_{\alpha} f\right\|_{L^{\Psi}\left(\mathbf{R}^{N}\right)} \leq C\|f\|_{L^{\Phi}\left(\mathbf{R}^{N}\right)} \leq C$,

$$
\left\|I_{\alpha} f\right\|_{L^{\Psi}(B(0,2))}+\int_{1}^{2}\left(\omega(r)\left\|I_{\alpha} f\right\|_{L^{\Psi}\left(\mathbf{R}^{N} \backslash B(0, r)\right)}\right)^{q} \frac{d r}{r} \leq C .
$$

TheOrem 10. Suppose $\Phi(x, t)$ satisfies $(\Phi 3 ; 0 ; p), \quad(\Phi 3 ; \infty ; q), \quad(\Phi 5 ; \eta)$, $(\Phi 6 ; \tau)$ and $(\Phi \alpha)$ for $p>1, q>1, \eta>0$ and $\tau>0$ satisfying $\eta \leq q / N$ and $\tau \leq p$. Assume that $\Psi(x, t)$ satisfies $(\Psi \Phi \alpha)$. For an integer $k \geq 1$, assume $\Phi_{\infty}(t)$ satisfies $\left(\Phi_{\infty} 2^{\prime}\right),\left(\Phi_{\infty} \omega 1 ; k-\alpha\right)$ and $\left(\Phi_{\infty} \omega 2 ; k-1-\alpha\right)$. Then there exists a constant $C>0$ such that

$$
\left\|I_{\alpha, k} f\right\|_{\mathscr{H}^{\Psi, q, \omega}\left(\mathbf{R}^{N}\right)} \leq C\|f\|_{\mathscr{H}^{\Phi, q, \omega}\left(\mathbf{R}^{N}\right)}
$$

for all $f \in \underline{\mathscr{H}}^{\Phi, q, \omega}\left(\mathbf{R}^{N}\right)$. 
Proof. Let $f$ be a nonnegative measurable function on $\mathbf{R}^{N}$ such that $\|f\|_{\mathscr{H}^{\Phi, q, \omega}\left(\mathbf{R}^{N}\right)} \leq 1$. Noting that

$$
\left|I_{\alpha, k}\left(f \chi_{B(0,4 r) \backslash B(0,|x| / 2) \backslash B(0,1)}\right)\right| \leq C I_{\alpha}\left(f \chi_{B(0,4 r) \backslash B(0,1)}\right)
$$

for $r \geq 1$ and $|x|<r$, by the same arguments as to obtain (18) in the proof of Theorem 5, we have

$$
\begin{aligned}
I_{\alpha, k} f(x) \leq C\{ & I_{\alpha}\left(f \chi_{B(0,4 r)}\right)(x) \\
& +r^{-\varepsilon_{2}^{\prime}+\alpha} \omega(r)^{-1} \Phi_{\infty}^{-1}\left(r^{-N}\right)\left(\int_{1 / 2}^{r}\left(t^{\varepsilon_{2}^{\prime}} \omega(t)\|f\|_{L^{\Phi}(A(0, t))}\right)^{q} \frac{d t}{t}\right)^{1 / q} \\
& \left.+r^{\varepsilon_{1}^{\prime}+\alpha} \omega(r)^{-1} \Phi_{\infty}^{-1}\left(r^{-N}\right)\left(\int_{r}^{\infty}\left(t^{-\varepsilon_{1}^{\prime}} \omega(t)\|f\|_{L^{\Phi}(A(0, t))}\right)^{q} \frac{d t}{t}\right)^{1 / q}\right\}
\end{aligned}
$$

for $r \geq 1$ and $x \in B(0, r)$, with $0<\varepsilon_{1}^{\prime}<\varepsilon_{1}$ and $0<\varepsilon_{2}^{\prime}<\varepsilon_{2}$.

Now, by Lemma 12

$$
\left\|I_{\alpha}\left(f \chi_{B(0,4 r)}\right)\right\|_{L^{\Psi}(B(0, r))} \leq C\|f\|_{L^{\Phi}(B(0,4 r))} .
$$

Thus, in the same way as in the proof of Theorem 4 (with $A(0, r)$ replaced by $B(0, r))$, we obtain

$$
\int_{1}^{\infty}\left(\omega(r)\left\|I_{\alpha, k} f\right\|_{L^{\Psi}(B(0, r))}\right)^{q} \frac{d r}{r} \leq C
$$

\section{Variable exponent H-M-M-O spaces}

Let $q(r)$ be a measurable function on $[1, \infty)$ satisfying

(Q1) $0<q^{-}:=\operatorname{ess~inf~}_{r \in[1, \infty)} q(r) \leq$ ess $\sup _{r \in[1, \infty)} q(r)=: q^{+}<\infty$.

Given $\Phi(x, t), \omega(r)$ and $q(r)$ as above, we denote by $\mathscr{H}^{\Phi, q(\cdot), \omega}\left(\mathbf{R}^{N}\right)$, $\underline{\mathscr{H}}^{\Phi, q(\cdot), \omega}\left(\mathbf{R}^{N}\right)$ and $\overline{\mathscr{H}}^{\Phi, q(\cdot), \omega}\left(\mathbf{R}^{N}\right)$ the classes of locally integrable functions $f$ on $\mathbf{R}^{N}$ satisfying

$$
\begin{gathered}
\|f\|_{\mathscr{H}^{\Phi, q(\cdot), \omega}\left(\mathbf{R}^{N}\right)}=\|f\|_{L^{\Phi}(B(0,2))}+\|\omega(\cdot)\| f\left\|_{L^{\Phi}(A(0, \cdot))}\right\|_{L^{q(\cdot)}((1, \infty), d r / r)}<\infty, \\
\|f\|_{\mathscr{H}^{\Phi, q(\cdot), \omega}\left(\mathbf{R}^{N}\right)}=\|\omega(\cdot)\| f\left\|_{L^{\Phi}(B(0, \cdot))}\right\|_{L^{q(\cdot)}((1, \infty), d r / r)}<\infty
\end{gathered}
$$

and

$$
\|f\|_{\overline{\mathscr{H}}^{\Phi, q(\cdot), \omega}\left(\mathbf{R}^{N}\right)}=\|f\|_{L^{\Phi}(B(0,2))}+\|\omega(\cdot)\| f\left\|_{L^{\Phi}\left(\mathbf{R}^{N} \backslash B(0, \cdot)\right)}\right\|_{L^{q(\cdot)}((1, \infty), d r / r)}<\infty
$$


respectively, where

$$
\|g\|_{L^{q(\cdot)}((1, \infty), d r / r)}=\inf \left\{\lambda>0 ; \int_{1}^{\infty}\left(\frac{|g(r)|}{\lambda}\right)^{q(r)} \frac{d r}{r} \leq 1\right\} .
$$

Proposition 2. Suppose $q(r)$ satisfies

(Q2) there exists a constant $q(\infty) \in(0, \infty)$ such that

$$
|q(r)-q(\infty)| \leq \frac{C_{q, \infty}}{\log (e+r)}
$$

whenever $r \geq 1$ with a constant $C_{q, \infty} \geq 0$.

Then

$$
\begin{aligned}
\mathscr{H}^{\Phi, q(\infty), \omega}\left(\mathbf{R}^{N}\right) & =\mathscr{H}^{\Phi, q(\cdot), \omega}\left(\mathbf{R}^{N}\right), \\
\underline{\mathscr{H}}^{\Phi, q(\infty), \omega}\left(\mathbf{R}^{N}\right) & =\underline{\mathscr{H}}^{\Phi, q(\cdot), \omega}\left(\mathbf{R}^{N}\right)
\end{aligned}
$$

and

$$
\overline{\mathscr{H}}^{\Phi, q(\infty), \omega}\left(\mathbf{R}^{N}\right)=\overline{\mathscr{H}}^{\Phi, q(\cdot), \omega}\left(\mathbf{R}^{N}\right) .
$$

ProOF. We only prove that $\mathscr{H}^{\Phi, q(\cdot), \omega}\left(\mathbf{R}^{N}\right) \subset \mathscr{H}^{\Phi, q(\infty), \omega}\left(\mathbf{R}^{N}\right)$, since the remaining assertions can be proved similarly. Let $f$ be a measurable function on $\mathbf{R}^{N}$ satisfying $\|f\|_{\mathscr{H}^{\Phi, q(\cdot), \omega}\left(\mathbf{R}^{N}\right)} \leq 1$. Then note that there exists a constant $c>0$ such that

$$
\int_{1 / \sqrt{2}}^{\infty}\left(\omega(r)\|f\|_{L^{\Phi}(A(0, r))}\right)^{q(r)} \frac{d r}{r} \leq c .
$$

First we show that

$$
\omega(r)\|f\|_{L^{\Phi}(A(0, r))} \leq C \quad \text { for } r \geq 1 .
$$

Let $J(r)=\omega(r)\|f\|_{L^{\Phi}(B(0, \sqrt{2} r) \backslash B(0, r))}$. If $r / \sqrt{2} \leq t \leq r$, then $B(0, \sqrt{2} r) \backslash B(0, r) \subset$ $A(0, t)$, so that

$$
J(r) \leq c_{1} c_{2} \omega(t)\|f\|_{L^{\Phi}(A(0, t))}
$$

by $(\omega 2)$. For $r \geq 1$, if $J(r) \geq c_{1} c_{2}$, then

$$
c \geq \int_{r / \sqrt{2}}^{r}\left(\omega(t)\|f\|_{L^{\Phi}(A(0, t))}\right)^{q(t)} \frac{d t}{t} \geq \frac{\log 2}{2}\left(c_{1}^{-1} c_{2}^{-1} J(r)\right)^{q^{-}},
$$

which implies

$$
J(r) \leq c_{1} c_{2}(2 c / \log 2)^{1 / q^{-}}
$$

Therefore, 


$$
\begin{aligned}
\omega(r)\|f\|_{L^{\Phi}(A(0, r))} & =\omega(r)\|f\|_{L^{\Phi}(B(0, \sqrt{2} r) \backslash B(0, r))}+\omega(r)\|f\|_{L^{\Phi}(B(0,2 r) \backslash B(0, \sqrt{2} r))} \\
& \leq J(r)+c_{1} c_{2} J(\sqrt{2} r) \leq C,
\end{aligned}
$$

which shows (25).

If $r^{-1}<\omega(r)\|f\|_{L^{\Phi}(A(0, r))}$, then we have by (Q2)

$$
\left(\omega(r)\|f\|_{L^{\Phi}(A(0, r))}\right)^{q(\infty)} \leq C\left(\omega(r)\|f\|_{L^{\Phi}(A(0, r))}\right)^{q(r)}
$$

for $r \geq 1$, which gives

$$
\begin{aligned}
& \int_{1}^{\infty}\left(\omega(r)\|f\|_{L^{\Phi}(A(0, r))}\right)^{q(\infty)} \frac{d r}{r} \\
& \quad \leq C \int_{1}^{\infty}\left(\omega(r)\|f\|_{L^{\Phi}(A(0, r))}\right)^{q(r)} \frac{d r}{r}+\int_{1}^{\infty} r^{-q(\infty)} \frac{d r}{r} \leq C .
\end{aligned}
$$

Thus, we obtain the required result.

By this proposition, Theorems 1, 2, 3, 4 and 5 are valid with $\mathscr{H}^{\Phi, q, \omega}\left(\mathbf{R}^{N}\right)$ replaced by $\mathscr{H}^{\Phi, q(\cdot), \omega}\left(\mathbf{R}^{N}\right)$, provided that $q(r)$ satisfies $\left(\mathrm{Q}_{2}\right)$, namely we have the following corollaries.

Corollary 1. Assume that $q(r)$ satisfies $(\mathrm{Q} 2)$. Suppose that $\Phi(x, t)$ satisfies $(\Phi 3 ; 0 ; p),(\Phi 3 ; \infty ; q),(\Phi 5 ; \eta)$ and $(\Phi 6 ; \tau)$ for $p>1, q>1, \eta>0$ and $\tau>0$ satisfying $\eta \leq q / N$ and $\tau \leq p$. Assume that $\Phi_{\infty}(t)$ satisfies $\left(\Phi_{\infty} 2\right)$, $\left(\Phi_{\infty} \omega 1 ; 0\right)$ and $\left(\Phi_{\infty} \omega 2 ;-N\right)$. Then the maximal operator $M$ is bounded from $\mathscr{H}^{\Phi, q(\cdot), \omega}\left(\mathbf{R}^{N}\right)$ to itself.

Corollary 2. Assume that $q(r)$ satisfies (Q2). For a real number $\beta$, suppose that $\Phi(x, t)$ satisfies $(\Phi 3 ; \infty ; q)$ and $(\Phi 5 ; \eta)$ for $q \geq 1$ and $\eta>0$ satisfying $\eta \leq q / N$. Assume that $\Phi(x, t)$ satisfies $(\Phi \omega ; \beta)$ and $\Phi_{\infty}(t)$ satisfies $\left(\Phi_{\infty} 2\right)$ and $\left(\Phi_{\infty} \omega 1 ; \beta\right)$. Then there exists a constant $C>0$ such that

$$
\left\|\hat{H}_{\beta}^{\infty} f\right\|_{\mathscr{H}^{\Phi, q(\cdot), \omega}\left(\mathbf{R}^{N}\right)} \leq C\|f\|_{\mathscr{H}^{\Phi, q(\cdot), \omega}\left(\mathbf{R}^{N}\right)}
$$

for all $f \in \mathscr{H}^{\Phi, q(\cdot), \omega}\left(\mathbf{R}^{N}\right)$.

COROllary 3. Assume that $q(r)$ satisfies (Q2). For a real number $\beta$, suppose that $\Phi(x, t)$ satisfies $(\Phi 3 ; \infty ; q)$ and $(\Phi 5 ; \eta)$ for $q \geq 1$ and $\eta>0$ satisfying $\eta \leq q / N$. Assume that $\Phi(x, t)$ satisfies $(\Phi \omega ; \beta)$ and $\Phi_{\infty}(t)$ satisfies $\left(\Phi_{\infty} 2\right)$ and $\left(\Phi_{\infty} \omega 2 ; \beta\right)$. Then there exists a constant $C>0$ such that

$$
\left\|\hat{H}_{\beta}^{0} f\right\|_{\mathscr{H}^{\Phi, q(\cdot), \omega}\left(\mathbf{R}^{N}\right)} \leq C\|f\|_{\mathscr{H}^{\Phi, q(\cdot), \omega}\left(\mathbf{R}^{N}\right)}
$$

for all $f \in \mathscr{H}^{\Phi, q(\cdot), \omega}\left(\mathbf{R}^{N}\right)$. 
COROllary 4. Assume that $q(r)$ satisfies (Q2). Suppose $\Phi(x, t)$ satisfies $(\Phi 3 ; 0 ; p),(\Phi 3 ; \infty ; q),(\Phi 5 ; \eta),(\Phi 6 ; \tau)$ and $(\Phi \alpha)$ for $p>1, q>1, \eta>0$ and $\tau>0$ satisfying $\eta \leq q / N$ and $\tau \leq p$. Assume that $\Psi(x, t)$ satisfies $(\Psi \Phi \alpha)$. For the function $\Phi_{\infty}(t)$, assume $\left(\Phi_{\infty} 2^{\prime}\right),\left(\Phi_{\infty} \omega 1 ;-\alpha\right)$ and $\left(\Phi_{\infty} \omega 2 ;-N\right)$ hold. Then there exists a constant $C>0$ such that

$$
\left\|I_{\alpha} f\right\|_{\mathscr{H}^{\Psi, q(\cdot), \omega}\left(\mathbf{R}^{N}\right)} \leq C\|f\|_{\mathscr{H}^{\Phi, q(\cdot), \omega}\left(\mathbf{R}^{N}\right)}
$$

for all $f \in \mathscr{H}^{\Phi, q(\cdot), \omega}\left(\mathbf{R}^{N}\right)$.

Corollary 5. Assume that $q(r)$ satisfies $(\mathrm{Q} 2)$. Suppose $\Phi(x, t)$ satisfies $(\Phi 3 ; 0 ; p),(\Phi 3 ; \infty ; q),(\Phi 5 ; \eta),(\Phi 6 ; \tau)$ and $(\Phi \alpha)$ for $p>1, q>1, \eta>0$ and $\tau>0$ satisfying $\eta \leq q / N$ and $\tau \leq p$. Assume that $\Psi(x, t)$ satisfies $(\Psi \Phi \alpha)$. For an integer $k \geq 1$, assume $\Phi_{\infty}(t)$ satisfies $\left(\Phi_{\infty} 2^{\prime}\right),\left(\Phi_{\infty} \omega 1 ; k-\alpha\right)$ and $\left(\Phi_{\infty} \omega 2\right.$; $k-1-\alpha)$. Then there exists a constant $C>0$ such that

$$
\left\|I_{\alpha, k} f\right\|_{\mathscr{H}^{\Psi, q(\cdot), \omega}\left(\mathbf{R}^{N}\right)} \leq C\|f\|_{\mathscr{H}^{\Phi, q(\cdot), \omega}\left(\mathbf{R}^{N}\right)}
$$

for all $f \in \mathscr{H}^{\Phi, q(\cdot), \omega}\left(\mathbf{R}^{N}\right)$.

Also, Theorems 6,8 and 10 hold with $\underline{\mathscr{H}}^{\Phi, q, \omega}\left(\mathbf{R}^{N}\right)$ replaced by $\underline{\mathscr{H}}^{\Phi, q(\cdot), \omega}\left(\mathbf{R}^{N}\right)$, and Theorems 7 and 9 hold with $\overline{\mathscr{H}}^{\Phi, q, \omega}\left(\mathbf{R}^{N}\right)$ replaced by $\frac{\overline{\mathscr{H}}}{\Phi, q(\cdot), \omega}\left(\mathbf{R}^{N}\right)$, when $q(r)$ satisfies $(\mathrm{Q} 2)$.

\section{Acknowledgement}

We would like to express our thanks to the referee for his/her comments.

\section{References}

[1] D. R. Adams, A note on Riesz potentials, Duke Math. J. 42 (1975), 765-778.

[2] D. R. Adams and L. I. Hedberg, Function Spaces and Potential Theory, Springer, 1996.

[3 ] A. Almeida and D. Drihem, Maximal, potential and singular type operators on Herz spaces with variable exponents, J. Math. Anal. Appl. 394 (2012), no. 2, 781-795.

[4] A. Almeida, J. Hasanov and S. Samko, Maximal and potential operators in variable exponent Morrey spaces, Georgian Math. J. 15 (2008), 195-208.

[5] P. Baroni, M. Colombo and G. Mingione, Harnack inequalities for double phase functionals, Nonlinear Anal. 121 (2015), 206-222.

[6] P. Baroni, M. Colombo and G. Mingione, Non-autonomous functionals, borderline cases and related function classes, St Petersburg Math. J. 27 (2016), 347-379.

[7] P. Baroni, M. Colombo and G. Mingione, Regularity for general functionals with double phase, Calc. Var. Partial Differential Equations 57 (2018), no. 2, Paper No. 62, 48 pp.

[8] B. Bojarski and P. Hajłasz, Pointwise inequalities for Sobolev functions and some applications, Studia Math. 106(1) (1993), 77-92. 
[9] V. I. Burenkov, A. Gogatishvili, V. S. Guliyev and R. Ch. Mustafayev, Boundedness of the fractional maximal operator in local Morrey-type spaces, Complex Var. Elliptic Equ. 55 (2010), no. 8-10, 739-758.

[10] V. I. Burenkov, A. Gogatishvili, V. S. Guliyev and R. Ch. Mustafayev, Boundedness of the Riesz potential in local Morrey-type spaces, Potential Anal. 35 (2011), no. 1, 67-87.

[11] F. Chiarenza and M. Frasca, Morrey spaces and Hardy-Littlewood maximal function, Rend. Mat. 7 (1987), 273-279.

[12] I. Chlebicka, A pocket guide to nonlinear differential equations in Musielak-Orlicz spaces, Nonlinear Anal. 175 (2018), 1-27.

[13] M. Colombo and G. Mingione, Regularity for Double Phase Variational Problems, Arch. Ration. Mech. Anal. 215 (2015), no. 2, 443-496.

[14] M. Colombo and G. Mingione, Bounded minimizers of double phase variational integrals, Arch. Rat. Mech. Anal. 218 (2015), 219-273.

[15] D. Cruz-Uribe and A. Fiorenza, Variable Lebesgue spaces. Foundations and harmonic analysis. Applied and Numerical Harmonic Analysis. Birkhauser/Springer, Heidelberg, 2013.

[16] D. Cruz-Uribe, A. Fiorenza and C. J. Neugebauer, The maximal function on variable $L^{p}$ spaces, Ann. Acad. Sci. Fenn. Math. 28 (2003), 223-238; Ann. Acad. Sci. Fenn. Math. 29 (2004), 247-249.

[17] L. Diening, Maximal functions in generalized $L^{p(\cdot)}$ spaces, Math. Inequal. Appl. 7(2) (2004), 245-254.

[18] L. Diening, Riesz potentials and Sobolev embeddings on generalized Lebesgue and Sobolev spaces $L^{p(\cdot)}$ and $W^{k, p(\cdot)}$, Math. Nachr. 263 (2004), 31-43.

[19] L. Diening, P. Harjulehto, P. Hästö and M. Růžička, Lebesgue and Sobolev spaces with variable exponents, Lecture Notes in Mathematics, 2017, Springer, Heidelberg, 2011.

[20] Z. Fu, Y. Lin and S. Lu, $\lambda$-central BMO estimates for commutators of singular integral operators with rough kernels, Acta Math. Sin. (Engl. Ser.) 24 (2008), no. 3, 373-386.

[21] A. Gogatishvili and R. Ch. Mustafayev, Dual spaces of local Morrey-type spaces, Czechoslovak Math. J. 61(136) (2011), no. 3, 609-622.

[22] V. S. Guliyev, J. J. Hasanov and S. G. Samko, Boundedness of the maximal, potential and singular operators in the generalized variable exponent Morrey spaces, Math. Scand. 107 (2010), no. 2, 285-304.

[23] V. S. Guliyev, J. J. Hasanov and S. G. Samko, Boundedness of maximal, potential type, and singular integral operators in the generalized variable exponent Morrey type spaces, Problems in mathematical analysis. No. 50. J. Math. Sci. (N. Y.) 170 (2010), no. 4, 423443.

[24] V. S. Guliyev, J. J. Hasanov and S. G. Samko, Maximal, potential and singular operators in the local "complementary" variable exponent Morrey type spaces, J. Math. Anal. Appl. 401 (2013), no. 1, 72-84.

[25] P. Harjulehto and P. Hästö, Boundary regularity under generalized growth conditions, Z. Anal. Anwend. 38 (2019), no. 1, 73-96.

[26] C. Herz, Lipschitz spaces and Bernstein's theorem on absolutely convergent Fourier transforms, J. Math. Mech. 18 (1968), 283-324.

[27] M. Izuki, Fractional integrals on Herz-Morrey spaces with variable exponent, Hiroshima Math. J. 40 (2010), no. 3, 343-355.

[28] J. L. Lewis, On very weak solutions of certain elliptic systems, Comm. Partial Differential Equations 18(9) (10) (1993), 1515-1537.

[29] X. Li and D. Yang, Boundedness of some sublinear operators on Herz spaces, Illinois J. Math. 40 (1996), 484-501. 
[30] F.-Y. Maeda, Y. Mizuta, T. Ohno and T. Shimomura, Boundedness of maximal operators and Sobolev's inequality on Musielak-Orlicz-Morrey spaces, Bull. Sci. Math. 137 (2013), 76-96.

[31] F.-Y. Maeda, Y. Mizuta, T. Ohno and T. Shimomura, Sobolev's inequality for double phase functionals, Forum. Math. 31 (2019), no. 2, 517-527.

[32] Y. Mizuta, Potential theory in Euclidean spaces, Gakkōtosho, Tokyo, 1996.

[33] Y. Mizuta, Integral representations, differentiability properties and limits at infinity for Beppo Levi functions, Potential Anal. 6 (1997), 237-276.

[34] Y. Mizuta, E. Nakai, T. Ohno and T. Shimomura, Riesz potentials and Sobolev embeddings on Morrey spaces of variable exponent, Complex Var. Elliptic Equ. 56 (2011), 671-695.

[35] Y. Mizuta and T. Ohno, Sobolev's theorem and duality for Herz-Morrey spaces of variable exponent, Ann. Acad. Sci. Fenn. Math. 39 (2014), 389-416.

[36] Y. Mizuta and T. Ohno, Herz-Morrey spaces of variable exponent, Riesz potential operator and duality, Complex Var. Elliptic Equ. 60 (2015), no. 2, 211-240.

[37] Y. Mizuta and T. Ohno, Boundedness of the maximal operator and Sobolev's inequality on non-homogeneous central Herz-Morrey-Orlicz spaces, Nonlinear Anal. 128 (2015), 325-347.

[38] Y. Mizuta, T. Ohno and T. Shimomura, Boundedness of maximal operators and Sobolev's theorem for non-homogeneous central Morrey spaces of variable exponent, Hokkaido Math. J. 44 (2015), 185-201.

[39] Y. Mizuta and T. Shimomura, Sobolev embeddings for Riesz potentials of functions in Morrey spaces of variable exponent, J. Math. Soc. Japan 60 (2008), 583-602.

[40] C. B. Morrey, On the solutions of quasi-linear elliptic partial differential equations, Trans. Amer. Math. Soc. 43 (1938), 126-166.

[41] J. Musielak, Orlicz spaces and modular spaces, Lecture Notes in Math. 1034, SpringerVerlag, 1983.

[42] E. Nakai, Hardy-Littlewood maximal operator, singular integral operators and the Riesz potentials on generalized Morrey spaces, Math. Nachr. 166 (1994), 95-103.

[43] E. Nakai, Calderón-Zygmund operators on Orlicz-Morrey spaces and modular inequalities, Banach and Function Spaces II, 393-410, Yokohama Publ., Yokohama, 2008.

[44] E. Nakai, Orlicz-Morrey spaces and the Hardy-Littlewood maximal function, Studia Math. 188 (2008), 193-221.

[45] T. Ohno and T. Shimomura, Boundedness of maximal operators and Sobolev's inequality on non-homogeneous central Musielak-Orlicz-Morrey spaces, Mediterr. J. Math. 13 (2016), no. 5, 3341-3357.

[46] J. Peetre, On the theory of $L_{p, \lambda}$ spaces, J. Funct. Anal. 4 (1969), 71-87.

[47] S. Samko, Variable exponent Herz spaces, Mediterr. J. Math. 10 (2013), no. 4, 2007-2025.

[48] A. Scapellato, Regularity of solutions to elliptic equations on Herz spaces with variable exponents, Bound. Value Probl. 2019, Paper No. 2, 9 pp.

[49] T. Shimomura and Y. Mizuta, Taylor expansion of Riesz potentials, Hiroshima Math. J. 25 (1995), 595-621.

[50] E. M. Stein, Singular integrals and differentiability properties of functions, Princeton Univ. Press, Princeton, 1970.

\author{
Fumi-Yuki Maeda \\ 4-24 Furue-higashi-machi, Nishi-ku \\ Hiroshima 733-0872, Japan \\ E-mail: fymaeda@h6.dion.ne.jp
}


Yoshihiro Mizuta

Department of Mathematics

Graduate School of Advanced Science and Engineering

Hiroshima University

Higashi-Hiroshima 739-8521, Japan

E-mail:yomizuta@hiroshima-u.ac.jp

Takao Ohno

Faculty of Education

Oita University

Dannoharu Oita-city 870-1192, Japan

E-mail: t-ohno@oita-u.ac.jp

Tetsu Shimomura

Department of Mathematics

Graduate School of Humanities and Social Sciences

Hiroshima University

Higashi-Hiroshima 739-8524, Japan

E-mail: tshimo@hiroshima-u.ac.jp 